\title{
Weakly coupled parametrically forced oscillator networks: existence, stability, and symmetry of solutions
}

\author{
Per Danzl · Jeff Moehlis
}

Received: 17 March 2009 / Accepted: 22 July 2009 / Published online: 15 August 2009

(C) The Author(s) 2009. This article is published with open access at Springerlink.com

\begin{abstract}
In this paper, we discuss existence, stability, and symmetry of solutions for networks of parametrically forced oscillators. We consider a nonlinear oscillator model with strong 2:1 resonance via parametric excitation. For uncoupled systems, the 2:1 resonance property results in sets of solutions that we classify using a combinatorial approach. The symmetry properties for solution sets are presented as are the group operators that generate the isotropy subgroups. We then impose weak coupling and prove that solutions from the uncoupled case persist for small coupling by using an appropriate Poincaré map and the Implicit Function Theorem. Solution bifurcations are investigated as a function of coupling strength and forcing frequency using numerical continuation techniques. We find that the characteristics of the single oscillator system are transferred to the network under weak coupling. We explore interesting dynamics that emerge with larger coupling strength, including anti-synchronized chaos and unsynchronized chaos. A classification for the symmetry-breaking that occurs due to weak coupling is presented for a simple example network.
\end{abstract}

P. Danzl $(\varangle) \cdot$ J. Moehlis

Department of Mechanical Engineering,

University of California, Santa Barbara, CA 93106, USA

e-mail:pdanzl@engineering.ucsb.edu

J. Moehlis

e-mail: moehlis@engineering.ucsb.edu
Keywords Parametrically forced oscillators . Coupled nonlinear oscillators · Symmetry

\section{Introduction}

The scientific study of coupled oscillators started with Christian Huygens' observations in the seventeenth century of mutual synchronization of pendulum clocks connected by a beam $[4,15]$. More recently, it has been recognized that mutual synchronization of coupled oscillators-the adjustment of rhythms of oscillating systems due to their weak interactions-occurs in many biological systems, including neurons during epileptic seizures [30] and pacemaker cells in the human heart [19]. Coupled oscillators have also been studied in detail for technological systems, such as arrays of lasers and superconducting Josephson junctions: see [25, 27], and [28], a recent popular book on the topic, for many biological and technological examples of synchronization for coupled oscillators.

We classify as autonomous oscillators those for whom the stable oscillations occur for an autonomous dynamical system, that is one for which there are no explicit time-dependent terms in the evolution equation. For example, the oscillations might arise through a Hopf bifurcation. In the limit of weak coupling, it is possible to reduce the dynamics of coupled autonomous oscillators to a phase model, with a single variable describing the phase of each oscillator with 
respect to some reference state (see, e.g., $[7,13,17$, 33]). This typically leads to models for which the dynamics depend only on the phase differences between different oscillators.

It is possible to show that several types of phaselocked solutions, for which the phase of all oscillators increases at the same constant rate, are guaranteed to exist in the weak coupling limit for any generic coupling function when the coupling topology has appropriate symmetry properties $[1,6,9,24]$; for the case of identical all-to-all coupling for $N$ oscillators, these are (i) in-phase solution: all $N$ oscillators have the same phase; (ii) two-block solutions: there are two blocks of oscillators, one in which $p$ oscillators share the same phase, and one in which $N-p$ oscillators share the same phase; (iii) rotating-block solutions: for $N=m k$, there are $m$ blocks with $k$ oscillators in each block sharing the same phase, with neighboring blocks differing in phase by $2 \pi / m$; (iv) double-rotating-block solutions: for $N=m\left(k_{1}+k_{2}\right)$, there are two rotatingblock solutions, one with $m$ blocks with $k_{1}$ oscillators in each block sharing the same phase and with neighboring blocks differing in phase by $2 \pi / \mathrm{m}$, another with $m$ blocks with $k_{2}$ oscillators in each block sharing the same phase and with neighboring blocks differing in phase by $2 \pi / m$, where there is a phase difference $0<\phi<2 \pi / m$ between a block with $k_{1}$ oscillators and the closest phase-advanced block with $k_{2}$ oscillators.

On the other hand, we classify as non-autonomous oscillators those for whom the stable oscillations only occur for a non-autonomous dynamical system, that is one for which there are explicit time-dependent terms such as time-periodic forcing. We will focus on parametrically forced oscillators, which are nonautonomous oscillators for which the forcing enters as a time-varying system parameter. Coupled parametrically forced oscillators arise in MEMS $[5,8,18]$ and other application areas [2, 3, 12, 29], but have not received as much theoretical research attention as coupled autonomous oscillator systems. This paper represents the first step in developing a comprehensive theory of the dynamics of general weakly coupled non-autonomous oscillators, in the spirit of the theory of general weakly coupled autonomous oscillators described in $[1,6]$. We hope that such a theory will ultimately lead to novel sensing mechanisms using MEMS devices; for simplicity, here we will consider a model system which represents only a caricature of such devices.
Specifically, in this paper we describe interesting synchronization phenomena that are possible for coupled parametrically forced oscillators. For example, consider two uncoupled oscillators whose response is at half the frequency of the driving voltage, as is common for MEMS devices [31]. Both oscillators could identically lock to the forcing, or they could lock one forcing period apart-both situations are allowable due to a discrete time-translation symmetry for the problem. We will show that different combinations of these states will persist if the oscillators are weakly coupled, with stability inherited from the stability properties of the periodic orbits which exist for the uncoupled system. This paper is an extension of results investigated in [20], and is organized as follows.

In Sect. 2 we consider the dynamics of a specific single parametrically forced oscillator and show that the origin is a stable equilibrium in the absence of forcing, and that for certain ranges of forcing frequency there exists a stable periodic orbit. Then, in Sect. 3.1, we consider a set of $N$ uncoupled parametrically forced oscillators, identifying different periodic states for such systems. In Sect. 3.2, the isotropy subgroups are presented as well as examples of the group operations that generate them.

Section 4.1 shows that provided the periodic orbits for the uncoupled system are hyperbolic, there will be periodic orbits for the weakly coupled system close to the periodic states identified for the uncoupled system. We prove this fact using an appropriate Poincaré map and the Implicit Function Theorem. In Sect. 4.2, we investigate the $N=2$ case in the presence of bi-directional coupling. We explore the bifurcation structure of solutions with respect to both coupling strength and forcing frequency. Interesting dynamics, such as anti-synchronized chaos, are found when the magnitude of the coupling strength is increased. Sect. 4.3 provides a detailed analysis of the $N=3$ case in the presence of bidirectional coupling for both the ring topology and the line topology. We perform a set of numerical bifurcation analyses to show how the magnitude and stability of the periodic oscillations vary with forcing frequency. For the $N=3$ case, we classify how the symmetry present in the uncoupled solution is either preserved or destroyed due to coupling for a simple example system for both the ring and the line topologies. We then relate the two topologies using an intermediate morphing topology (a ring with a single variable connection) and discuss 
Fig. 1 Bifurcation diagram for fixed $b=0.2$ and $F=0.5$. Solid (resp., dashed) lines indicate stable (resp., unstable) solutions

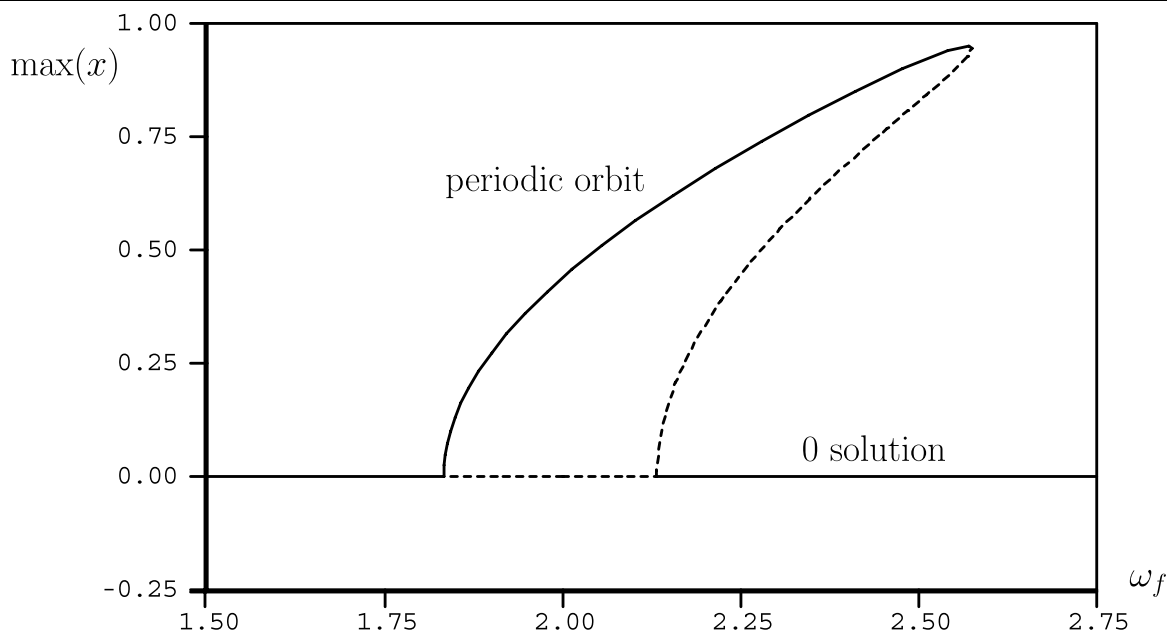

how symmetry-related solutions organize differently depending on the network structure. We describe how these results can be generalized to $N$ coupled parametrically forced oscillators in Sect. 4.4. Finally, we give conclusions in Sect. 5.

\section{A parametrically forced oscillator}

Consider the equation for a damped, parametrically forced oscillator

$\ddot{x}+b \dot{x}+x+x^{3}=x F \cos \left(\omega_{f} t\right)$.

Here the term $b \dot{x}$ represents damping (we assume $b>0$ ), the term $x+x^{3}$ represents a nonlinear restoring force, and the term $x F \cos \left(\omega_{f} t\right)$ represents parametric excitation which can be viewed as a time-periodic modulation of the linear part of the restoring force. For this system, if $F=0$ then $x \rightarrow 0$ as $t \rightarrow \infty$, as follows. Letting

$V(x, \dot{x})=\frac{1}{2} x^{2}+\frac{1}{4} x^{4}+\frac{1}{2} \dot{x}^{2}$,

we find that

$\frac{d V}{d t}=-b \dot{x}^{2} \leq 0$,

with equality only if $\dot{x}=0$. The only point in phase space which starts in the set of points for which $V(x, \dot{x})=0$ and remains in this set for all time is $(x, \dot{x})=(0,0)$; by the LaSalle Invariance Prin- ciple [32], all trajectories thus approach this point as $t \rightarrow \infty$.

For appropriate $F$ and $\omega_{f}$, the system has a periodic response. Indeed, treating $\omega_{f}$ as a bifurcation parameter for fixed $F$ and $b$, we obtain the bifurcation diagram shown in Fig. 1. (This and other numerical bifurcation analyses were done using AUTO [10] or XPP [11].) The 0 solution is characterized by $x=\dot{x}=$ 0 for all time; it exists for all $\omega_{f}$, being unstable near $\omega_{f}=2$ and stable otherwise for the range shown. It loses stability in a bifurcation to a periodic orbit, with the periodic orbit branch turning around in a saddlenode bifurcation so that there is a region of bistability between the periodic orbit and the 0 solution. Such a bifurcation structure is common for MEMS devices, see e.g. [26, 34].

Figure 2 indicates the types of dynamics which occur in different parameter ranges, with the "parabola" corresponding to the loss of stability of the 0 solution, and the "straight line" corresponding to the saddle-node bifurcation of the periodic orbit branch. As shown in Fig. 3, the response of this periodic orbit is at half the frequency of the forcing, as is common for parametrically forced oscillators [23]. We note that an equally valid periodic orbit for this forcing is shown in Fig. 4, which is shifted by one period of the forcing from the solution shown in Fig. 3; clearly these solutions are related by a time-translation symmetry. Indeed, our example oscillator governed by (1) has a very convenient form of discrete time-translation symmetry which is also related to phase space symmetry: translating by one forcing period is equivalent to multiplying both states at time $t$ by -1 , so $(x(t+T)$, 
Fig. 2 Existence and stability of solutions for different regions of parameter space for $b=0.2$, with bifurcations sets shown as lines
Fig. 3 Stable periodic orbit for $b=0.2, F=0.5$, $\omega_{f}=2$. The response is at half the frequency of the forcing. We will refer to this as the $A$ solution
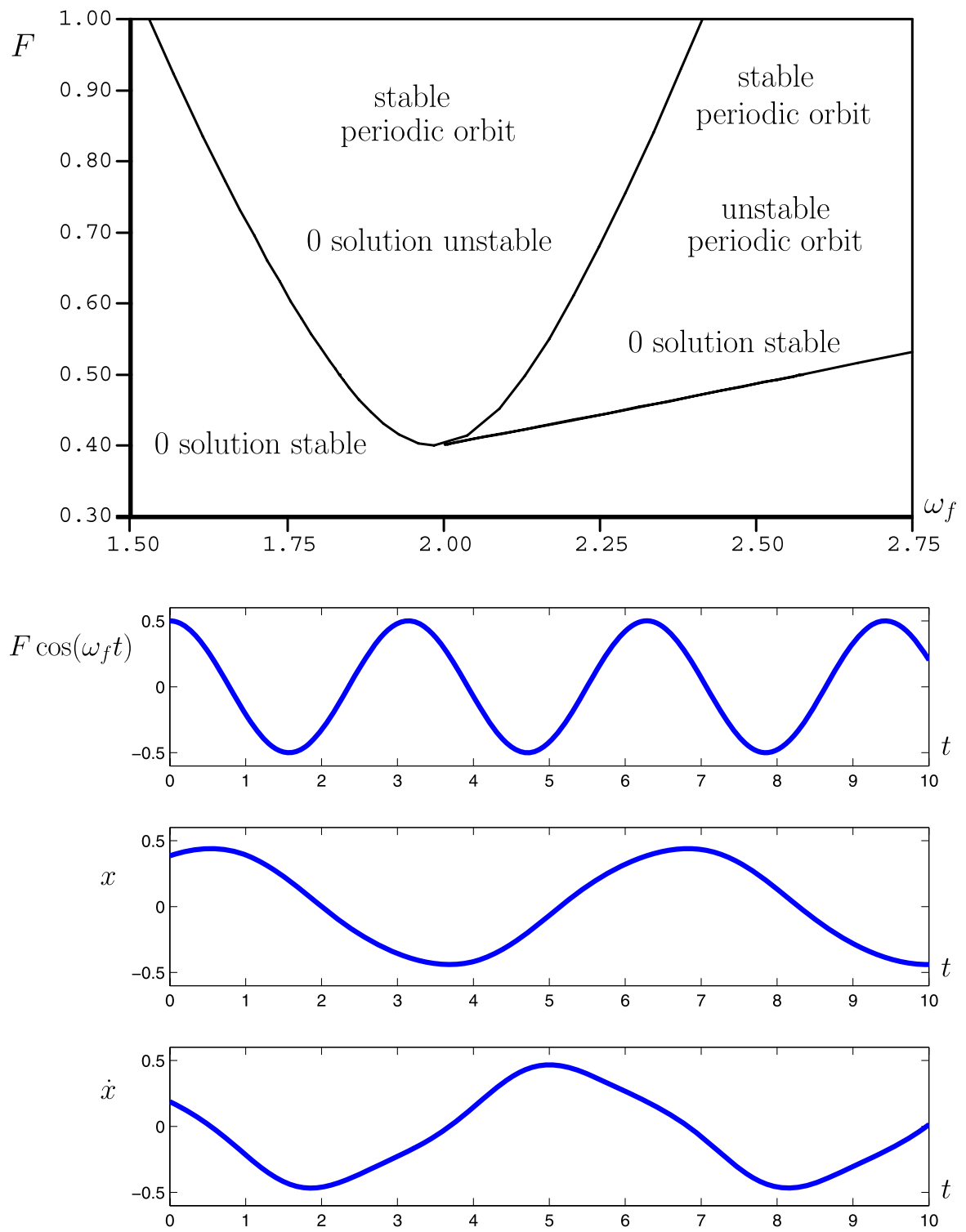

$\dot{x}(t+T))=(-x(t),-\dot{x}(t))$. These symmetry-related solutions will be crucial for understanding the different possible solutions when such oscillators are weakly coupled. We will find it convenient to distinguish the solutions shown in Figs. 3 and 4 by referring to one of them as the $A$ solution and the other as the $B$ solution. We introduce a new solution label $z \in\{A, B, 0\}$. The $A$ and $B$ solutions have the same $\max (x)$ value, and the periodic orbit branch in Fig. 1 corresponds to both (symmetry-related) solutions. The $z=0$ label corresponds to the previously defined 0 solution, $x=\dot{x}=0$. This notation will greatly simplify the following presentation.

\section{Uncoupled parametrically forced oscillators}

\subsection{Combinatorial considerations}

Suppose

$b=0.2, \quad F=0.5, \quad \omega_{f}=2$,

so that when $c=0$, each oscillator could be in a stable periodic state given by the $A$ and $B$ solutions, or it could be in the unstable 0 solution. There will be $3^{N}$ distinct periodic orbits for $c=0$ : oscillator 1 could be 
Fig. 4 Stable periodic orbit for $b=0.2, F=0.5$, $\omega_{f}=2$. This is shifted by one period of the forcing from the solution shown in Fig. 3. We will refer to this as the $B$ solution
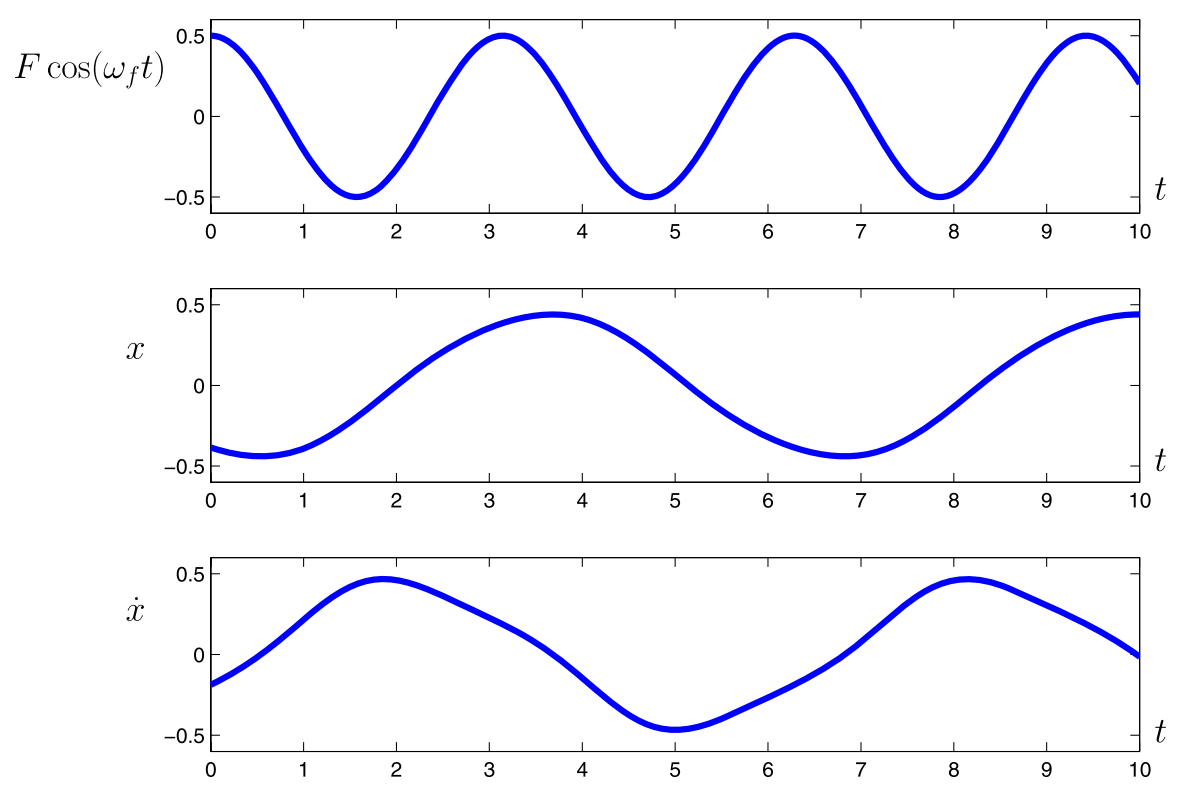

in $A, B$, or 0 , oscillator 2 could be in $A, B$, or 0 , etc. Of these solutions, a total of

$$
\frac{N !}{p_{A} ! p_{B} ! p_{0} !}
$$

solutions will have $p_{z}$ oscillators in each state $z=A$, $B$, or 0 , where $p_{A}+p_{B}+p_{0}=N$. This follows from the following combinatorial argument. Suppose we make a list of $N$ symbols such that the $i$ th symbol is $A, B$, or 0 according to whether the $i$ th oscillator is in the $A, B$, or 0 state, respectively. In the $N$ slots, there are

$$
\left(\begin{array}{c}
N \\
p_{A}
\end{array}\right)=\frac{N !}{p_{A} !\left(N-p_{A}\right) !}
$$

different ways to put the symbol $A$ in $p_{A}$ of the slots. Of the remaining $\left(N-p_{A}\right)$ slots, there are

$$
\left(\begin{array}{c}
N-p_{A} \\
p_{B}
\end{array}\right)=\frac{\left(N-p_{A}\right) !}{p_{B} !\left(N-p_{A}-p_{B}\right) !}
$$

different ways to put the symbol $B$ in $p_{B}$ of the slots. The remaining $\left(N-p_{A}-p_{B}\right)$ slots will have the symbol 0 . The product of these is

$\frac{N !}{p_{A} !\left(N-p_{A}\right) !} \times \frac{\left(N-p_{A}\right) !}{p_{B} !\left(N-p_{A}-p_{B}\right) !}=\frac{N !}{p_{A} ! p_{B} ! p_{0} !}$, as in (3). As an illustration, suppose $N=4, p_{A}=2$, $p_{B}=1$, and $p_{0}=1$. The different possible lists of symbols are

$\begin{array}{lll}A A B 0 & A A 0 B & A B A 0 \\ A 0 A B & A B 0 A & A 0 B A \\ B A A 0 & 0 A A B & B A 0 A \\ 0 A B A & B 0 A A & 0 B A A,\end{array}$

giving a total of

$\frac{4 !}{2 ! 1 ! 1 !}=12$

possibilities. This combinatorial argument readily generalizes to situations in which more than three states are possible for each oscillator, for example when $b=0.2, F=0.5$, and $\omega_{f}=2.25$.

\subsection{Symmetry considerations}

Periodic solution trajectories of an uncoupled $N$ oscillator system are described by the population labeling variable $\mathbf{z}$, a length- $N$ set of single oscillator solution labels $z_{i}$ for $i=1, \ldots, N$. Since the order of the entries is irrelevant for an uncoupled system, we can group any state string into successive substrings of $A$ 's, $B$ 's, and 0's. For example, a $00 A 0 A B 0 A B$ state is equivalent to an $A A A B B 0000$ state. In any state 
string, there are $N_{A} A$ 's, $N_{B} B$ 's, and $N_{0} 0$ 's, where $N_{A}+N_{B}+N_{0}=N$.

It is possible to classify solutions of dynamical systems with symmetry according to their isotropy subgroup, which is the subgroup of all symmetry operations which leave the solution unchanged. It is convenient to order the solution into the substrings of $A$ 's, $B$ 's, and 0's as described above. Then, we see that the isotropy subgroup of the system is

$S_{\left(N_{A}+N_{B}\right)} \times S_{N_{0}}$,

where

$S_{\left(N_{A}+N_{B}\right)}=\left\langle\kappa_{1}, \kappa_{2}\right\rangle, \quad S_{N_{0}}=\left\langle\kappa_{3}, \kappa_{4}\right\rangle$,

$\kappa_{1} \cdot\left(z_{1}, z_{2}, \ldots, z_{N_{A}-1}, z_{N_{A}}\right.$,

$z_{N_{A}+1}, z_{N_{A}+2}, \ldots, z_{N_{A}+N_{B}-1}, z_{N_{A}+N_{B}}$,

$\left.z_{N_{A}+N_{B}+1}, z_{N_{A}+N_{B}+2}, \ldots, z_{N-1}, z_{N}\right)$

$\rightarrow\left(z_{2}, z_{1}, \ldots, z_{N_{A}-1}, z_{N_{A}}\right.$,

$z_{N_{A}+1}, z_{N_{A}+2}, \ldots, z_{N_{A}+N_{B}-1}, z_{N_{A}+N_{B}}$,

$\left.z_{N_{A}+N_{B}+1}, z_{N_{A}+N_{B}+2}, \ldots, z_{N-1}, z_{N}\right)$,

$\kappa_{2} \cdot\left(z_{1}, z_{2}, \ldots, z_{N_{A}-1}, z_{N_{A}}\right.$,

$z_{N_{A}+1}, z_{N_{A}+2}, \ldots, z_{N_{A}+N_{B}-1}, z_{N_{A}+N_{B}}$,

$\left.z_{N_{A}+N_{B}+1}, z_{N_{A}+N_{B}+2}, \ldots, z_{N-1}, z_{N}\right)$

$\rightarrow\left(-z_{N_{A}+N_{B}}, z_{1}, z_{2}, \ldots, z_{N_{A}-1}\right.$,

$-z_{N_{A}}, z_{N_{A}+1}, z_{N_{A}+2}, \ldots, z_{N_{A}+N_{B}-1}$,

$\left.z_{N_{A}+N_{B}+1}, z_{N_{A}+N_{B}+2}, \ldots, z_{N-1}, z_{N}\right)$,

$\kappa_{3} \cdot\left(z_{1}, z_{2}, \ldots, z_{N_{A}-1}, z_{N_{A}}\right.$,

$z_{N_{A}+1}, z_{N_{A}+2}, \ldots, z_{N_{A}+N_{B}-1}, z_{N_{A}+N_{B}}$,

$\left.z_{N_{A}+N_{B}+1}, z_{N_{A}+N_{B}+2}, \ldots, z_{N-1}, z_{N}\right)$

$\rightarrow\left(z_{1}, z_{2}, \ldots, z_{N_{A}-1}, z_{N_{A}}\right.$,

$z_{N_{A}+1}, z_{N_{A}+2}, \ldots, z_{N_{A}+N_{B}-1}, z_{N_{A}+N_{B}}$,

$\left.z_{N_{A}+N_{B}+2}, z_{N_{A}+N_{B}+1}, \ldots, z_{N-1}, z_{N}\right)$,

$\kappa_{4} \cdot\left(z_{1}, z_{2}, \ldots, z_{N_{A}-1}, z_{N_{A}}\right.$,

$$
\begin{aligned}
& z_{N_{A}+1}, z_{N_{A}+2}, \ldots, z_{N_{A}+N_{B}-1}, z_{N_{A}+N_{B}}, \\
& \left.z_{N_{A}+N_{B}+1}, z_{N_{A}+N_{B}+2}, \ldots, z_{N-1}, z_{N}\right) \\
\rightarrow & \left(z_{1}, z_{2}, \ldots, z_{N_{A}-1}, z_{N_{A}},\right. \\
& z_{N_{A}+1}, z_{N_{A}+2}, \ldots, z_{N_{A}+N_{B}-1}, z_{N_{A}+N_{B}}, \\
& \left.z_{N}, z_{N_{A}+N_{B}+1}, z_{N_{A}+N_{B}+2}, \ldots, z_{N-1}\right) .
\end{aligned}
$$

In words, $\kappa_{1}$ swaps the first two $A$ solutions, $\kappa_{2}$ is a cyclic permutation of the $A$ and $B$ solutions with minus signs appropriately included, $\kappa_{3}$ swaps the first two 0 solutions, and $\kappa_{4}$ is a cyclic permutation of the 0 solutions. The intuition behind this result is that the solution is unchanged by permuting the first $N_{A}+N_{B}$ states (the $A$ and $B$ states) however one desires, provided the $A$ and $B$ states are transformed into each other through appropriate multiplications by -1 . The $N_{0} 0$ solutions can be permuted however desired without changing the solution.

Let us again consider our example system $A A A B B 0000$. This solution has isotropy subgroup $S_{5} \times S_{4}$, being unchanged by multiplication by any product of $\kappa_{1}, \kappa_{2}, \kappa_{3}, \kappa_{4}$. For example, it is unchanged under

$$
\begin{gathered}
\kappa_{3} \kappa_{2}^{2} \kappa_{1} \kappa_{2} \kappa_{1} \kappa_{2}^{3} \cdot\left(z_{1}, z_{2}, z_{3}, z_{4}, z_{5}, z_{6}, z_{7}, z_{8}, z_{9}\right) \\
\rightarrow\left(-z_{5}, z_{1},-z_{4},-z_{2},-z_{3}, z_{7}, z_{6}, z_{8}, z_{9}\right) .
\end{gathered}
$$

This discussion readily generalizes to conditions where the individual oscillators have more than three possible states by including additional $\kappa$ operators to swap and permute the additional possible states.

\section{Coupled parametrically forced oscillators}

\subsection{Periodic orbits for weakly coupled oscillators}

When $c \neq 0$ but is small, we expect solutions analogous to those for $c=0$ to exist, as follows. We write our system as

$\dot{\mathbf{x}}=\mathbf{f}(\mathbf{x}, t)+c \mathbf{g}(\mathbf{x})$,

where

$\mathbf{x}=\left(x_{1}, \dot{x}_{1}, \ldots, x_{N}, \dot{x}_{N}\right)^{T}$,

$\mathbf{f}(\mathbf{x}, t)$ captures the terms which are independent of $c$, and $\mathbf{g}(\mathbf{x})$ captures the coupling terms. Let $T=2 \pi / \omega_{f}$ be the period of the forcing. We define $P_{c}$ to be the time- $2 T$ map, that is, the map which takes an initial condition (the state at $t=0$ ) to the state obtained by evolving for a time equal to twice the period of the forcing. Now, let

$h(\mathbf{x}, c)=P_{c}(\mathbf{x})-\mathbf{x}$, 
and let $\mathbf{q}_{0}$ be a point on one of the periodic solutions of the uncoupled problem, for example the $A A$ solution in the $N=2$ case. We see that

$h\left(\mathbf{q}_{0}, 0\right)=0$.

The Implicit Function Theorem (see Appendix) then implies that, provided the matrix $D_{\mathbf{x}} h\left(\mathbf{q}_{0}, 0\right)$ is invertible, there is a unique solution $\mathbf{q}(c)$ close to $\mathbf{q}_{0}$, for any sufficiently small $c$, such that $h(\mathbf{q}(c), c)=0$. This implies that

$P_{c}(\mathbf{q}(c))=\mathbf{q}(c)$,

that is, $\mathbf{q}(c) \approx \mathbf{q}_{0}$ is a fixed point of the time- $2 T$ map, which means that it is a point on a periodic orbit with period $2 T$ which is close to a periodic orbit of the uncoupled system. (A related argument is used to prove part (ii) of Theorem 4.1.1 in [14].)

It is instructive to consider an alternative, but equivalent argument. We know that $\mathbf{q}_{0}$ is a fixed point of $P_{0}$, that is,

$P_{0}\left(\mathbf{q}_{0}\right)=\mathbf{q}_{0}$.

We will determine, to leading order in $c$, the condition which must be met for $\mathbf{q}(c)$ to be a fixed point of $P_{c}$. Consider the asymptotic expansions

$\mathbf{q}(c)=\mathbf{q}_{0}+c \mathbf{q}_{1}+\cdots$,

$P_{c}(\mathbf{x})=P_{0}(\mathbf{x})+c p_{1}(\mathbf{x})+\cdots$.

Setting $P_{c}(\mathbf{q}(c))=\mathbf{q}(c)$, we obtain

$$
\begin{aligned}
\mathbf{q}_{0} & +c \mathbf{q}_{1}+\cdots \\
& =P_{c}\left(\mathbf{q}_{0}+c \mathbf{q}_{1}+\cdots\right) \\
& =P_{0}\left(\mathbf{q}_{0}+c \mathbf{q}_{1}+\cdots\right)+p_{1}\left(\mathbf{q}_{0}+c \mathbf{q}_{1}+\cdots\right) \\
& =P_{0}\left(\mathbf{q}_{0}\right)+c D P_{0}\left(\mathbf{q}_{0}\right) \mathbf{q}_{1}+c p_{1}\left(\mathbf{q}_{0}\right)+\cdots .
\end{aligned}
$$

This is valid at $\mathcal{O}\left(c^{0}\right)$ from (10). At $\mathcal{O}\left(c^{1}\right)$, we need

$$
\mathbf{q}_{1}=D P_{0}\left(\mathbf{q}_{0}\right) \mathbf{q}_{1}+p_{1}\left(\mathbf{q}_{0}\right) .
$$

Solving for $\mathbf{q}_{1}$,

$\mathbf{q}_{1}=\left[I d-D P_{0}\left(\mathbf{q}_{0}\right)\right]^{-1} p_{1}\left(\mathbf{q}_{0}\right)$,

where $I d$ is the identity matrix. In order to solve for $\mathbf{q}_{1}$, it is necessary that $\left[I d-D P_{0}\left(\mathbf{q}_{0}\right)\right]$ be invertible.
This is equivalent to the above condition for the Implicit Function Theorem to hold that $D_{\mathbf{x}} h\left(\mathbf{q}_{0}, 0\right)$ be invertible.

We now show that this matrix is invertible provided the periodic orbit for the uncoupled system is hyperbolic. Suppose that $v$ is an eigenvector of $D P_{0}\left(\mathbf{q}_{0}\right)$ with eigenvalue $\lambda$, so that

$\left[D P_{0}\left(\mathbf{q}_{0}\right)\right] v=\lambda v$.

Then

$\left(I d-\left[D P_{0}\left(\mathbf{q}_{0}\right)\right]\right) v=(1-\lambda) v$.

Thus, the matrix $\left(I d-\left[D P_{0}\left(\mathbf{q}_{0}\right)\right]\right)$ only has a zero eigenvalues if $\lambda=1$. But the eigenvalues of $D P_{0}\left(\mathbf{q}_{0}\right)$ give the stability of the periodic orbit for the uncoupled problem; in particular, if it is a hyperbolic periodic orbit, none of the eigenvalues is on the unit circle. The hyperbolicity condition only needs to be checked for a single uncoupled oscillator, since we are assuming that the oscillators are identical.

Summarizing, provided the periodic orbit for the uncoupled system is hyperbolic, there will be a nearby periodic orbit for the system with sufficiently small coupling.

Furthermore, we expect that since the $c \rightarrow 0$ system limits to the $c=0$ system, the periodic orbit for the weakly coupled system will "inherit" the stability properties from the periodic orbit for the uncoupled system. This follows from the continuity of the Poincaré map with respect to $c$, giving

$\lim _{c \rightarrow 0} D P_{c}(\mathbf{q}(c))=D P_{0}\left(\mathbf{q}_{0}\right)$.

This implies that the eigenvalues corresponding to the stability of the $\mathbf{q}(c)$ periodic orbit for the coupled system tend toward the eigenvalues corresponding to the stability of the $\mathbf{q}_{0}$ periodic orbit for the uncoupled system.

\section{$4.2 N=2$ coupled oscillators}

Now consider $N=2$ parametrically forced oscillators which are coupled linearly:

$\ddot{x}_{1}+b \dot{x}_{1}+x_{1}+x_{1}^{3}=x_{1} F \cos \left(\omega_{f} t\right)+c\left(x_{2}-x_{1}\right)$,

$\ddot{x}_{2}+b \dot{x}_{2}+x_{2}+x_{2}^{3}=x_{2} F \cos \left(\omega_{f} t\right)+c\left(x_{1}-x_{2}\right)$,

where $x_{i}$ is the position of the $i$ th oscillator, $i=1,2$. 
If $c=0$, these are independent parametrically forced oscillators. Thus, for $b=0.2, F=0.5$, and $\omega_{f}=2$, each oscillator could be in a stable periodic state given by the $A$ or $B$ solutions; each oscillator also has an unstable 0 solution. The periodic solutions for the uncoupled two-oscillator system are given in Table 1.

Here the first symbol characterizes the state of the first oscillator, and the second symbol characterizes the state of the second oscillator. In-phase solutions have both oscillators responding identically to the forcing, while out-of-phase solutions correspond to each oscillator undergoing an oscillation which is shifted by one period of the forcing relative to the other. Large-small solutions have one oscillator undergoing oscillations locked to the forcing while the other oscillator is stationary. The name comes from

Table 1 Periodic solutions for uncoupled two-oscillator system

\begin{tabular}{ll}
\hline Type & Equivalent states \\
\hline In-phase & $A A, B B$ \\
Out-of-phase & $A B, B A$ \\
Large-small & $A 0, B 0,0 A, 0 B$ \\
00 solution & 00 \\
\hline
\end{tabular}

the result that when weak coupling is introduced, the former oscillator will undergo relatively large oscillations, while the latter will undergo relatively small oscillations. For the 00 solution, both oscillators are stationary. The distinct solutions within a given class (in-phase, out-of-phase, or large-small) are related by symmetry.

Results for $c \neq 0$ are illustrated for (18)-(19) for $b=0.2, F=0.5, \omega_{f}=2$ in Fig. 5, which show that for small $c>0$, periodic orbits of the expected stability type exist and are close to the periodic orbits for the uncoupled system. Indeed, for the uncoupled system the $A$ and $B$ solutions are stable and the 0 solution is unstable (see Fig. 1); thus, the $A A$ and $A B$ solutions (and their symmetry-related counterparts $B B$ and $B A$, respectively) are expected to be stable for small $|c|$, while the others are expected to be unstable. Figure 5 shows that as $c$ increases, the out-of-phase $A B$ solution loses stability. This illustrates that our arguments above are only valid for small $|c|$. We note that, in this figure, the fact that the branches come together at $c=0$ is an artifact of the projection. The solutions are actually separated in phase space: even though they share the same value for $\max \left(\left|x_{1}\right|\right)$, the second oscillator has different behavior. Therefore, the uniqueness property from the Implicit Function Theorem argument is not violated.

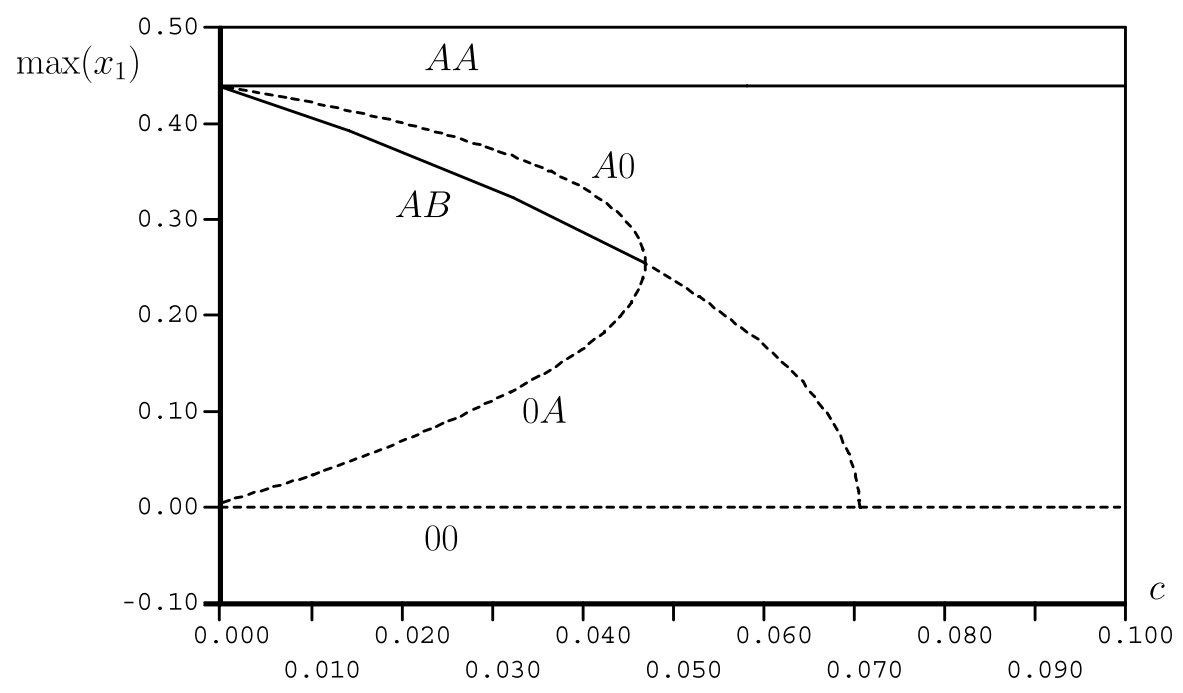

Fig. 5 Bifurcation diagram for fixed $b=0.2, F=0.5, \omega_{f}=2$ with the coupling strength $c$ treated as a bifurcation parameter. Solid (resp., dashed) lines indicate stable (resp., unstable) solutions. For small (positive) $c$, from top to bottom, the solutions of the branches approach the $A A, A 0, A B, 0 A$, and 00 as $c \rightarrow 0$.
Note, for example, that the $A A$ and $B B$ solutions have the same $\max \left(x_{1}\right)$ value, and thus appear to be on the same branch in this projection. A similar coincidence between symmetry-related solutions occurs for all other branches 
Fig. 6 Bifurcation diagram for fixed $b=0.2, F=0.5$, $\omega_{f}=2.25$ with the coupling strength $c$ treated as a bifurcation parameter. Solid (resp., dashed) lines indicate stable (resp., unstable) solutions

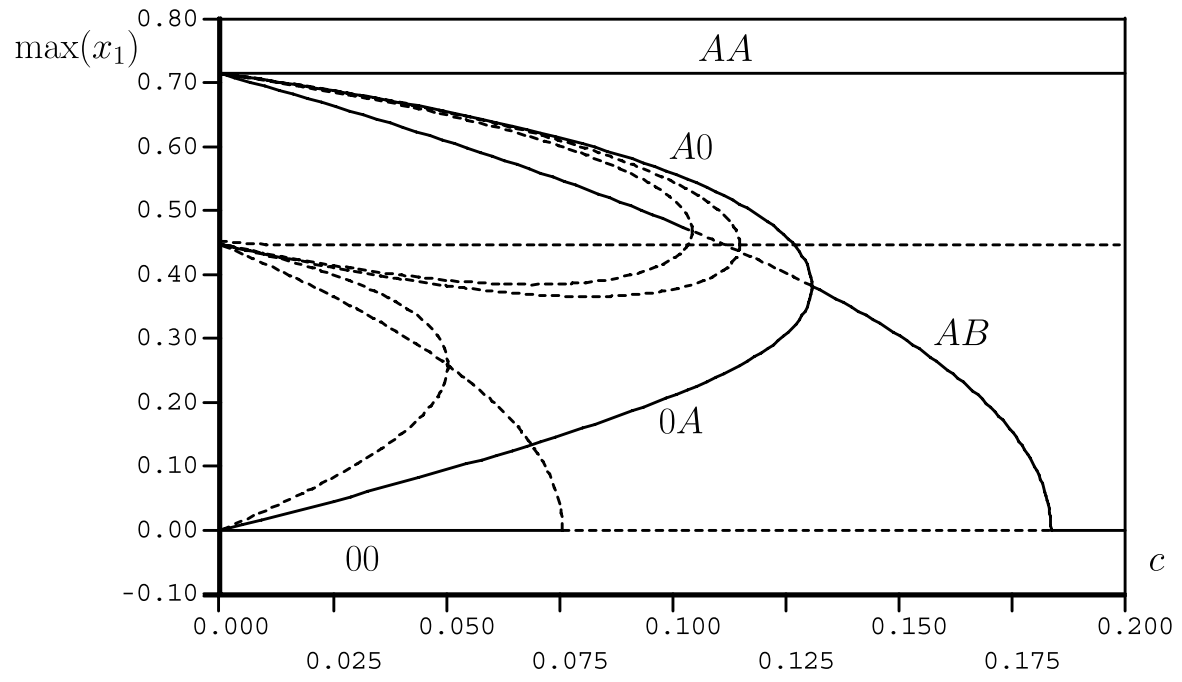

Fig. 7 Partial bifurcation diagram for (18)-(19) for fixed $b=0.2, F=0.5$, $c=0.03$ with $\omega_{f}$ treated as a bifurcation parameter. Solid (resp., dashed) lines indicate stable (resp., unstable) solutions

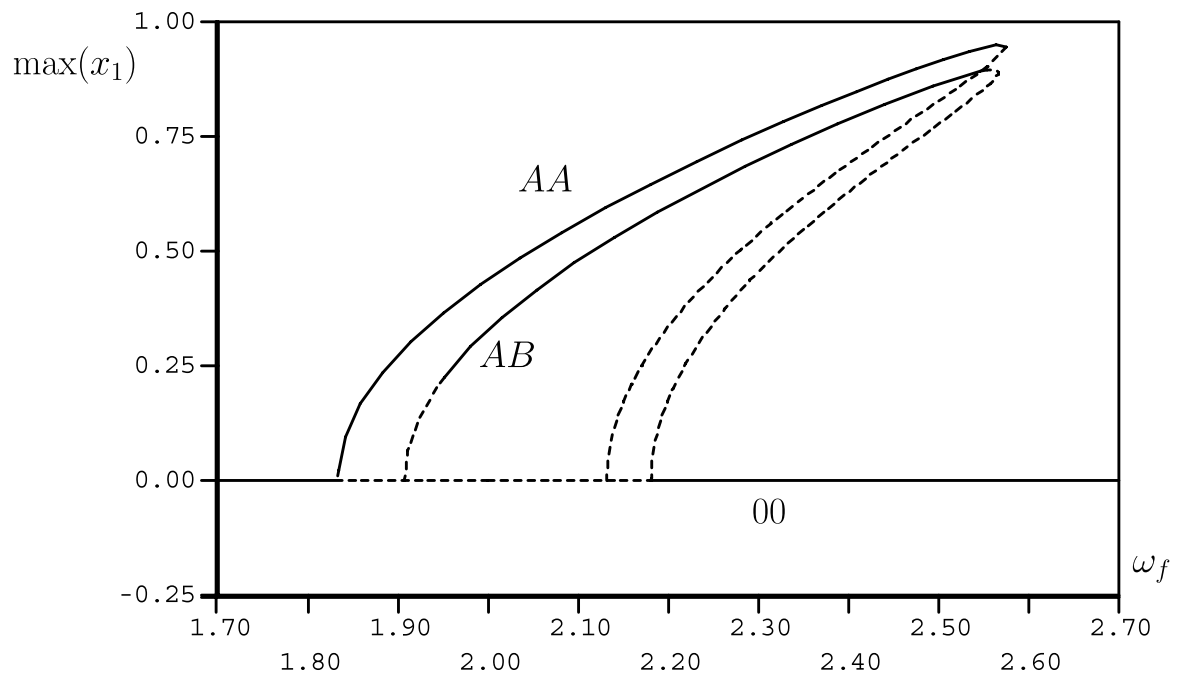

For $b=0.2, F=0.5, \omega_{f}=2.25$, Fig. 1 shows that the 0 solution is stable, and that there are stable periodic orbits (which are analogs of the $A$ and $B$ solutions discussed above) and unstable periodic orbits. Figure 6 shows that for small $c>0$, periodic orbits of the expected stability type exist and are close to the periodic orbits for the uncoupled system. (To aid in interpreting this plot, we note that the maximum $x$ values for the stable and unstable periodic orbits are approximately 0.72 and 0.45 , respectively.) Indeed, for the uncoupled system the $A, B$, and 0 solutions are stable; thus, the in-phase $A A$ solutions, out-of-phase $A B$ solutions, large-small solutions (which in the limit $c \rightarrow 0$ approach the $A 0$ solutions), and 00 solution are all stable for small $|c|$. All solutions which involve an unsta- ble periodic orbit for the uncoupled system as $c \rightarrow 0$ are unstable.

We now fix the coupling strength as $c=0.03$ and take $b=0.2, F=0.5$, and treat $\omega_{f}$ as a bifurcation parameter. Figure 7 shows the corresponding bifurcation diagram for the in-phase, out-of-phase, and 00 solutions. We see that the in-phase and out-of-phase solutions bifurcate from the 00 solution at different values of $\omega_{f}$. As expected from the discussion above, for $\omega_{f}=2$ both the in-phase and out-of-phase solutions are stable, while for $\omega_{f}=2.25$ the in-phase, out-ofphase, and 00 solutions are all stable. We expect at $\omega_{f}=2.25$ there will be large-small solutions; this is verified in Fig. 8 which identifies them as being on a branch which bifurcates from the out-of-phase solu- 
Fig. 8 Full bifurcation diagram for (18)-(19) for fixed $b=0.2, F=0.5$, $c=0.03$ with $\omega_{f}$ treated as a bifurcation parameter. Solid (resp., dashed) lines indicate stable (resp., unstable) solutions
Fig. 9 Example of a large-small periodic orbit for $N=2, b=0.2$, $F=0.5, \omega_{f}=2.25$, $c=0.03$. In the lower panel, the dark line and light line correspond to the two different oscillators
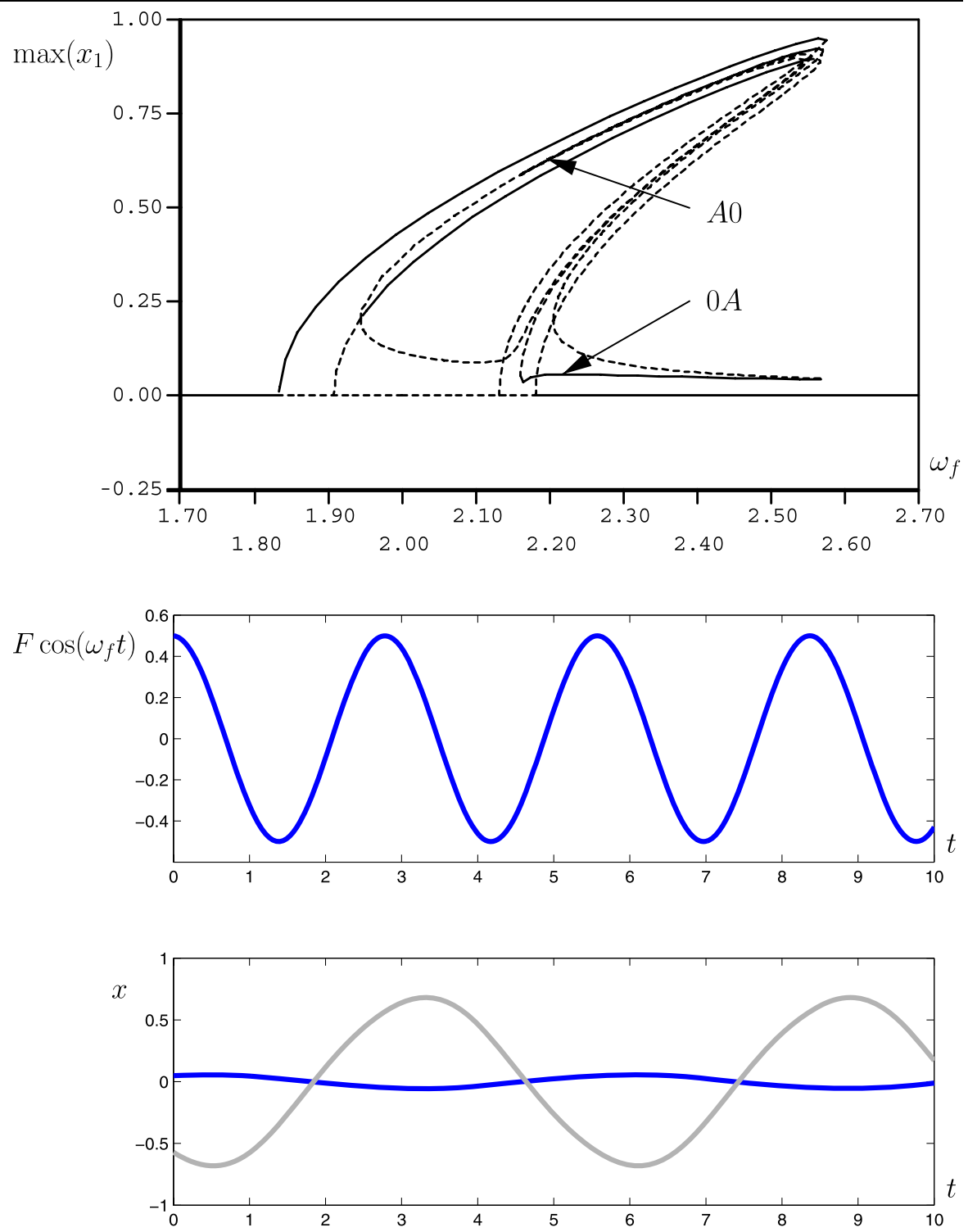

tion branch. The large-small solution at $\omega_{f}=2.25$ is shown in Fig. 9.

Although our analytical results only apply in the weak coupling limit, we note that interesting dynamics occur for larger $|c|$, such as anti-synchronized chaotic behavior for $b=0.2, F=0.5, \omega_{f}=2$, and $c=-0.9$ shown in Figs. 10 and 11.

\section{$4.3 N=3$ coupled oscillators}

For the identical bidirectional coupling considered in this paper, the $N=3$ case is the smallest network wherein network topology plays a significant role. We consider

$$
\begin{aligned}
\ddot{x}_{1} & +b \dot{x}_{1}+x_{1}+x_{1}^{3} \\
& =x_{1} F \cos \left(\omega_{f} t\right)+c\left(x_{2}-x_{1}\right)+\tilde{c}\left(x_{3}-x_{1}\right), \\
\ddot{x}_{2} & +b \dot{x}_{2}+x_{2}+x_{2}^{3} \\
& =x_{2} F \cos \left(\omega_{f} t\right)+c\left(x_{1}-x_{2}\right)+c\left(x_{3}-x_{2}\right), \\
\ddot{x}_{3} & +b \dot{x}_{3}+x_{3}+x_{3}^{3} \\
& =x_{3} F \cos \left(\omega_{f} t\right)+\tilde{c}\left(x_{1}-x_{3}\right)+c\left(x_{2}-x_{3}\right) .
\end{aligned}
$$

The cases $\tilde{c}=c$ and $\tilde{c}=0$ are referred to ring and line coupling topologies, respectively, and are shown 
Fig. 10 Time series for anti-synchronized chaos for $N=2, b=0.2, F=0.5$, $\omega_{f}=2$, and $c=-0.9$, where the dark line and light line correspond to the two different oscillators

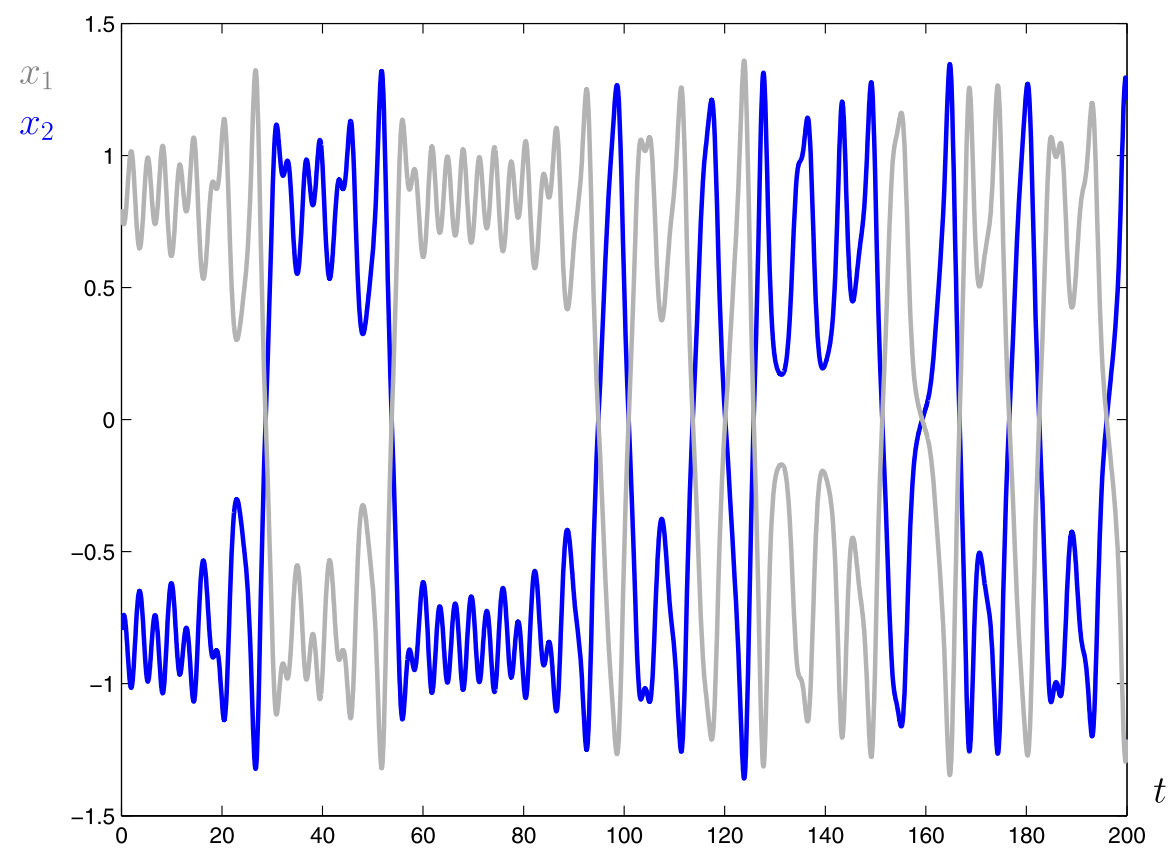

Fig. 11 Phase space representation of anti-synchronized chaos for $N=2, b=0.2, F=0.5$, $\omega_{f}=2$, and $c=-0.9$, where the dark line and light line correspond to the two different oscillators

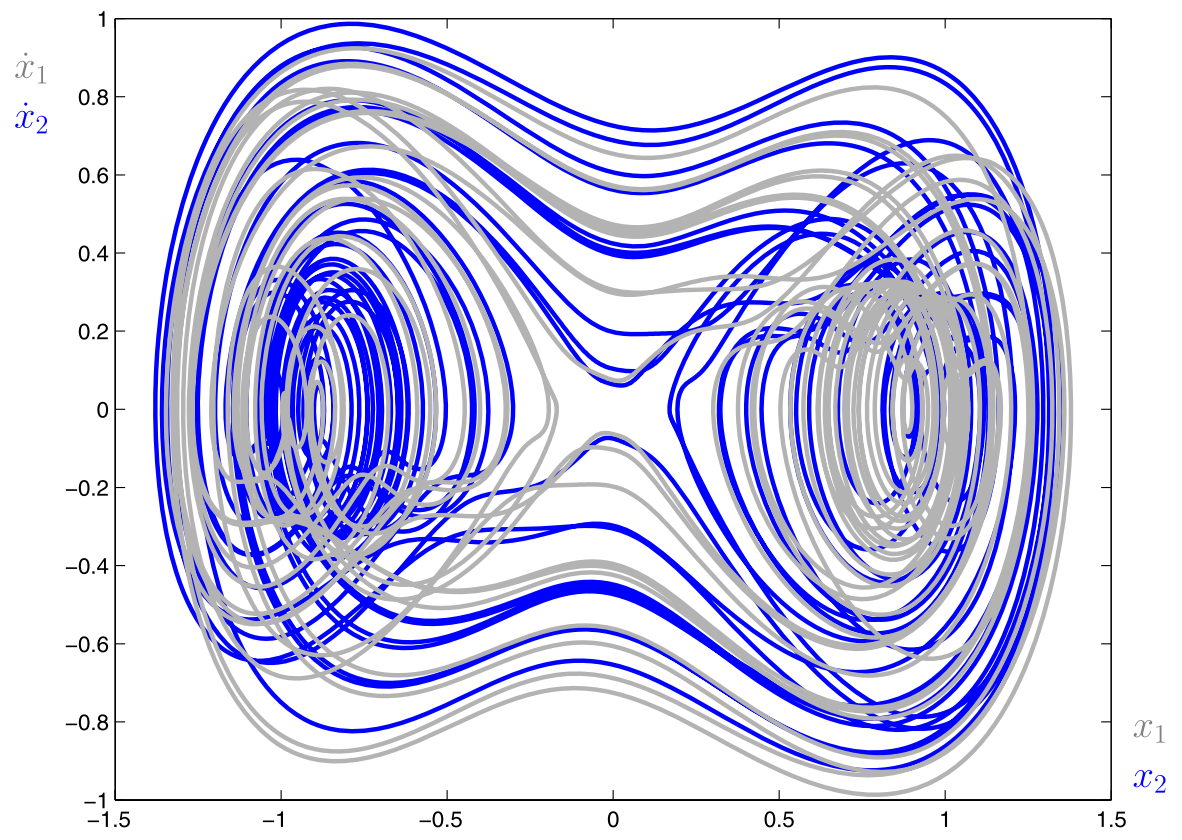

in Fig. 12. We will consider the limit of weak coupling and we will show how the network topology will partition the set of all possible solutions into sets of symmetry-related solutions. We note that, due to the form of coupling, there will always exist a zero solution 000 with stability inherited from the uncoupled case, and a class of stable synchronous solutions
$\{A A A, B B B\}$, regardless of network topology. In the following analysis, we will be specifically considering the case where $b=0.2, \omega_{f}=2, F=0.5$, which corresponds to the region in bifurcation space where, for a single oscillator, only the 0 solution and the large periodic orbit exist. The numerics were done using AUTO [10], which enables the analysis of stable and unsta- 


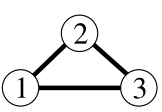

(a)

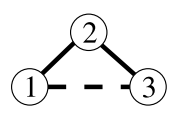

(b)

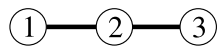

(c)
Fig. $12 N=3$ oscillator networks. Ring $[\tilde{c}=c](\mathbf{a})$, morph $[0<\tilde{c}<c](b)$, and line $[\tilde{c}=0](\mathbf{c})$

ble orbits. We note that the representative solutions we consider are particular to the system described by (20)-(22).

\subsubsection{Notation}

For convenience, we will introduce additional notation to describe the solution types and indicate solution symmetries. The $z \in\{A, B, 0\}$ solution label may now be indexed by subscripts, i.e. $z_{X^{Y}}$. The $X$ subscript variable indicates two different qualifications of the solution, depending on the $z$ solution type. If the solution is near or identically zero, i.e. $z=0$, the subscript $X \in\{A, B, 0\}$ indicates if the solution is identically zero $(X=0)$ or if it is a small (near-zero) periodic orbit with phase the same as either the $A$ or $B$ large periodic orbits ( $X=A$ or $X=B$, respectively). For solutions near or identically zero, the $Y$ superscript on the $X$ subscript is not used.

For the large periodic orbit states $z \in\{A, B\}$, the subscript $X \in\{L, S, 0\}$ denotes the relative size of the solution and is intended to provide a qualification of the symmetry when coupling is included. If the solution is identical to the uncoupled stable periodic orbit, we will denote this by $X=0$. If, on the other hand, the solution's magnitude (i.e. $\max _{t \in(0, T]}(x(t))$ ) is larger (resp., smaller) than the uncoupled stable periodic orbit, we denote this by $X=L$ (resp., $X=S$ ). When two or more oscillators both exhibit a large periodic orbit close in magnitude to the uncoupled solution, we will use our notation to indicate symmetry. For example, if there are two identical $A$ solutions that are larger than the uncoupled orbit, we will indicate this by $A_{L} A_{L}$. If there are two $A$ solutions that are larger than the uncoupled solution, but not equal to each other, we will indicate this using the $Y$ superscript as $A_{L^{+}} A_{L^{-}}$. The $Y \in\{-,+\}$ superscript will be used with the $0_{X}$ labels as well, also to indicate non-identical orbits. The ' + ' solution is so marked because it has a larger magnitude than the '-' solution (again, quantifying magnitude by $\left.\max _{t \in(0, T]}(x(t))\right)$. It is worthwhile to note here that
Table $2 N=3$ ring symmetry classes

\begin{tabular}{lll}
\hline Label & Symmetry-related states & Stability \\
\hline$R_{1}$ & 000 & Unstable \\
$R_{2}$ & $A A A, B B B$ & Stable \\
$R_{3}$ & $A A 0, A 0 A, 0 A A, B B 0, B 0 B, 0 B B$ & Unstable \\
$R_{4}$ & $A A B, A B A, B A A, B B A, B A B, A B B$ & Stable \\
$R_{5}$ & $A 00,0 A 0,00 A, B 00,0 B 0,00 B$ & Unstable \\
$R_{6}$ & $A B 0, A 0 B, B 0 A, 0 B A, 0 A B, B A 0$ & Unstable \\
\hline
\end{tabular}

these orbits and their magnitudes are all well-defined in the limit of small $|c|$.

For the rest of our discussion, when we refer to oscillators being synchronized, we mean phasesynchrony, which does not imply any relationship between amplitudes. When amplitudes are equal, we will use the terms identical if they are indistinguishable, or anti-synchronized if they are in anti-phase.

\subsubsection{Ring topology}

For the $N=3$ case, the ring topology is the same as the all-to-all topology. Each oscillator is coupled identically to the others. Even in such a simple network as shown below, the dynamics are rich, both for small coupling and as the coupling strength becomes largely negative.

The ring topology gives six symmetry classes including the zero $\left(R_{1}\right)$ and synchronous $\left(R_{2}\right)$ solutions, which are summarized in Table 2.

The $R_{3}$ class is the set of all solutions that have two oscillators with synchronous large periodic orbits and one with a small near-zero magnitude orbit. For $c \lesssim 0$, the large periodic orbits are slightly larger in magnitude than the uncoupled orbit and the near-zero orbit is in phase. For $0 \lesssim c$, the two synchronous large periodic orbits are slightly smaller than the uncoupled orbit, and the near-zero orbit is out of phase. This solution is depicted in Fig. 13. All solutions in the $R_{3}$ class are unstable for small $|c|$.

The $R_{4}$ class consists of all solutions wherein two oscillators have synchronized large periodic orbits and the other oscillator has a similar large periodic orbit, but shifted in time by one forcing frequency. With nonzero coupling, the $S_{3}$ symmetry of the $A A B$ solution is broken. For $c \lesssim 0$, the two synchronous periodic orbits are smaller in magnitude than the anti-synchronous orbit, resulting in an $S_{2}$ symmetry. The relative magnitudes of the periodic orbits switch for the positive 
Fig. 13 Example of $R_{3}$ $F=0.5, \omega_{f}=2.25$, $c=0.03$ with ring topology. In the lower panel, the dark line, the light line, and the dashed line correspond to the three different oscillators, the latter two of which have identical solutions periodic orbit for $b=0.2$,
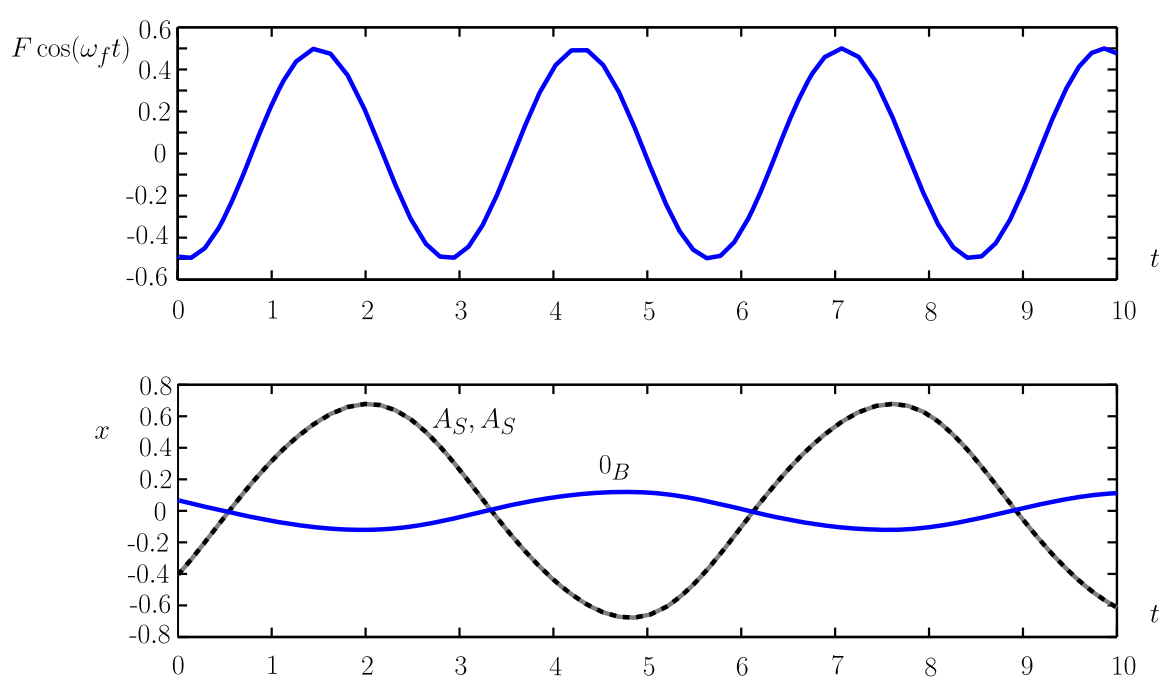

Fig. 14 Example of $R_{4}$ periodic orbit for $b=0.2$, $F=0.5, \omega_{f}=2.25$, $c=0.03$ with ring topology. In the lower panel, the dark line, the light line, and the dashed line correspond to the three different oscillators, the latter two of which have identical solutions
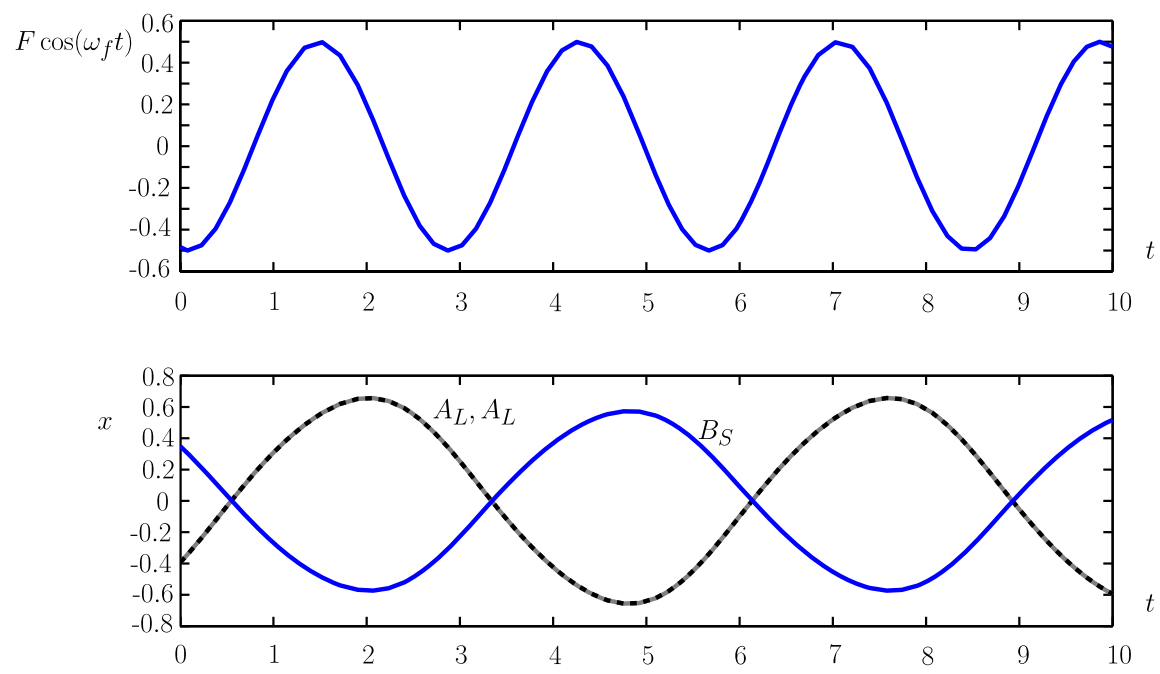

small coupling case, $0 \lesssim c$, shown in Fig. 14 . The $R_{4}$ solutions are all stable for small $|c|$.

The $R_{5}$ class is the set of all solutions that have a single oscillator with a large periodic orbit and two oscillators with near-zero orbits. For $c \lesssim 0$, the large periodic orbit is larger than the uncoupled orbit and the two near-zero solutions are identical (retaining an analog of the symmetry of the uncoupled solution) and in phase with the other oscillator. As $c$ is increased through zero, the magnitude of the $A$ solution becomes smaller than the uncoupled orbit, and the two nearzero solutions switch to become out of phase with the $A$ solution (but still synchronous and identical to each other). All solutions in the $R_{5}$ class are unstable for small $|c|$.
The $R_{6}$ class contains all solutions that have two oscillators with anti-synchronized large periodic orbits while the third oscillator has a near-zero orbit. For $c \lesssim 0$, the two large periodic orbits have identical magnitudes and are slightly larger than the uncoupled solution. For $0 \lesssim c$, the two large periodic orbits are have identical magnitudes and are slightly smaller than the uncoupled solution. In all the cases, the near-zero solution is, in fact, identically zero. Thus, the symmetry present in the uncoupled solution is preserved for sets of solutions in the $R_{6}$ class. All solutions in the $R_{6}$ class are unstable for small $|c|$.

The properties of the ring topology solutions for $c=0$ and $|c| \approx 0$ are summarized in Tables 2 and 3 . 
Fig. $15 N=3$ ring topology bifurcation diagram for fixed $b=0.2$, $F=0.5, c=0.03$ with $\omega_{f}$ treated as a bifurcation parameter. Solid (resp., dashed) lines indicate stable (resp., unstable) solutions. Stable solutions are labeled by symmetry class per the $0 \lesssim c$ column of Table 3, and have symmetry-related solutions tabulated in Table 2

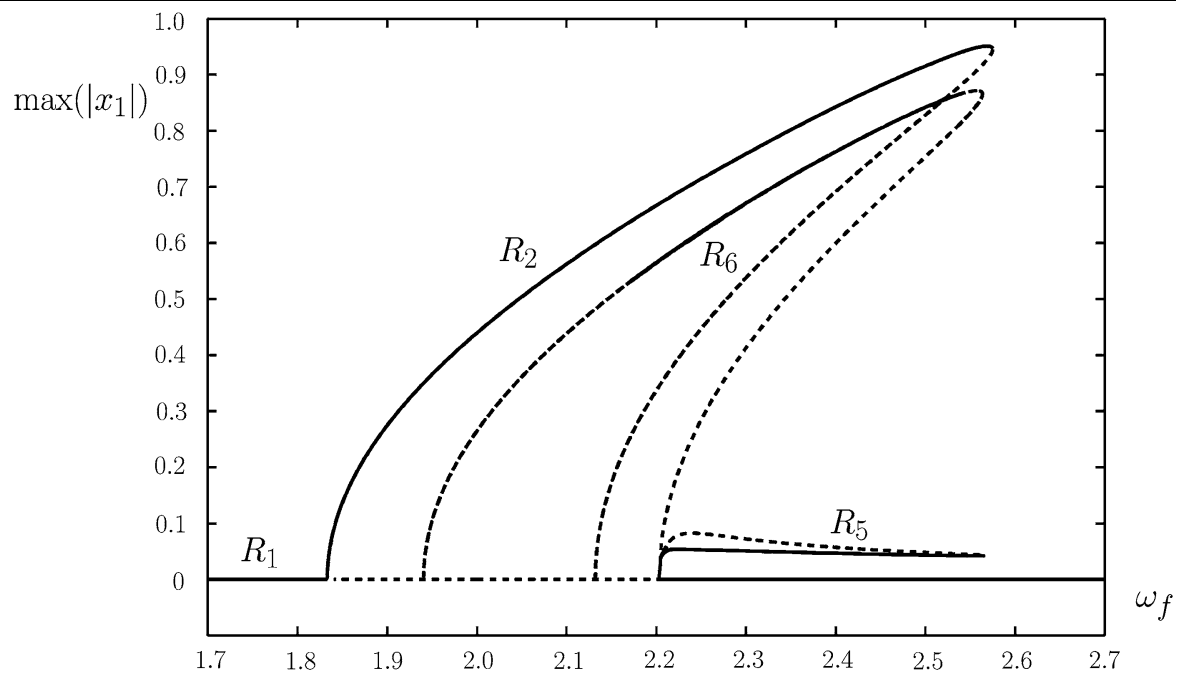

Table 3 Solution representatives for $|c| \approx 0$ for each symmetry class for ring topology

\begin{tabular}{llll}
\hline Symmetry class & $c \lesssim 0$ & $c=0$ & $0 \lesssim c$ \\
\hline$R_{1}$ & $0_{0} 0_{0} 0_{0}$ & $0_{0} 0_{0} 0_{0}$ & $0_{0} 0_{0} 0_{0}$ \\
$R_{2}$ & $A_{0} A_{0} A_{0}$ & $A_{0} A_{0} A_{0}$ & $A_{0} A_{0} A_{0}$ \\
$R_{3}$ & $A_{L} A_{L} 0_{A}$ & $A_{0} A_{0} 0_{0}$ & $A_{S} A_{S} 0_{B}$ \\
$R_{4}$ & $A_{S} A_{S} B_{L}$ & $A_{0} A_{0} B_{0}$ & $A_{L} A_{L} B_{S}$ \\
$R_{5}$ & $A_{L} 0_{A} 0_{A}$ & $A_{0} 0_{0} 0_{0}$ & $A_{S} 0_{B} 0_{B}$ \\
$R_{6}$ & $A_{L} B_{L} 0_{0}$ & $A_{0} B_{0} 0_{0}$ & $A_{S} B_{S} 0_{0}$ \\
\hline
\end{tabular}

\subsubsection{Forcing-frequency bifurcation diagram: Ring topology}

We again fix the coupling strength as $c=0.03$ and take $b=0.2, F=0.5$, and treat $\omega_{f}$ as a bifurcation parameter. Figure 15 shows the $\max \left(\left|x_{1}\right|\right)$-projection of the corresponding bifurcation diagram. This projection is identical in appearance to the $\max \left(\left|x_{2}\right|\right)$ and $\max \left(\left|x_{3}\right|\right)$ bifurcation diagrams due to the label permutation symmetry of the ring topology. The in-phase $A A A$ solution and the $A 0 B$ solution bifurcate from the 000 solution at different values of $\omega_{f}$. This bifurcation diagram is similar to the $N=2$ case shown in Figs. 7 and 8 . The main features are preserved, such as the large-small solution, which is now a large-smallsmall solution (or in our new notation, $0_{A} 0_{A} B$ ).

\subsubsection{Chaotic dynamics}

Similarly to the $N=2$ case, interesting dynamics also emerge as $|c|$ is increased. Figure 16 shows un- synchronized chaotic behavior for $b=0.2, F=0.5$, $\omega_{f}=2$, and $c=-0.9$. The phase portrait of the $N=3$ chaotic system shown in Fig. 17 displays a doublewell potential structure similar to that of the $N=2$ case shown previously in Fig. 11, although the trajectories of the three oscillators are not synchronized.

\subsubsection{Line topology}

The line topology breaks the symmetry of the ring and introduces new symmetry classes, which are summarized in Table 4. There still exists an unstable zero solution $\left(L_{1}\right)$ and a stable synchronous solution $\left(L_{2}\right)$. However, the line topology means that the "center" oscillator gets input from both of the two "end" oscillators, while the end oscillators only receive input from center oscillator. This has dramatic effect on the symmetry class partitions.

The $L_{3}$ class represents solutions where the center oscillator is synchronized in a large periodic orbit with one of the end oscillators. The other end oscillator is near-zero. Solutions in this class exhibit interesting bifurcation behavior. One of the end oscillators retains the uncoupled orbit amplitude for all sufficiently small $c$, regardless of sign. This can be interpreted as a region of the amplitude versus coupling strength curve where the slope is zero. For $c \lesssim 0$, the center oscillator has a periodic orbit that is slightly larger in amplitude than the uncoupled orbit and the other end oscillator has an in-phase near-zero orbit. For small positive coupling, the center oscillator has a slightly smaller magnitude orbit, and the near-zero orbit of the other end 
Fig. 16 Time series for unsynchronized chaos for $N=3$ ring with $b=0.2$, $F=0.5, \omega_{f}=2$, and $c=-0.9$, where the dark line, the dashed line, and light line correspond to the three different oscillators
Fig. 17 Phase space representation of unsynchronized chaos for $N=3$ ring with $b=0.2$, $F=0.5, \omega_{f}=2$, and $c=-0.9$, where the dark line, the dashed line, and light line correspond to the three different oscillators
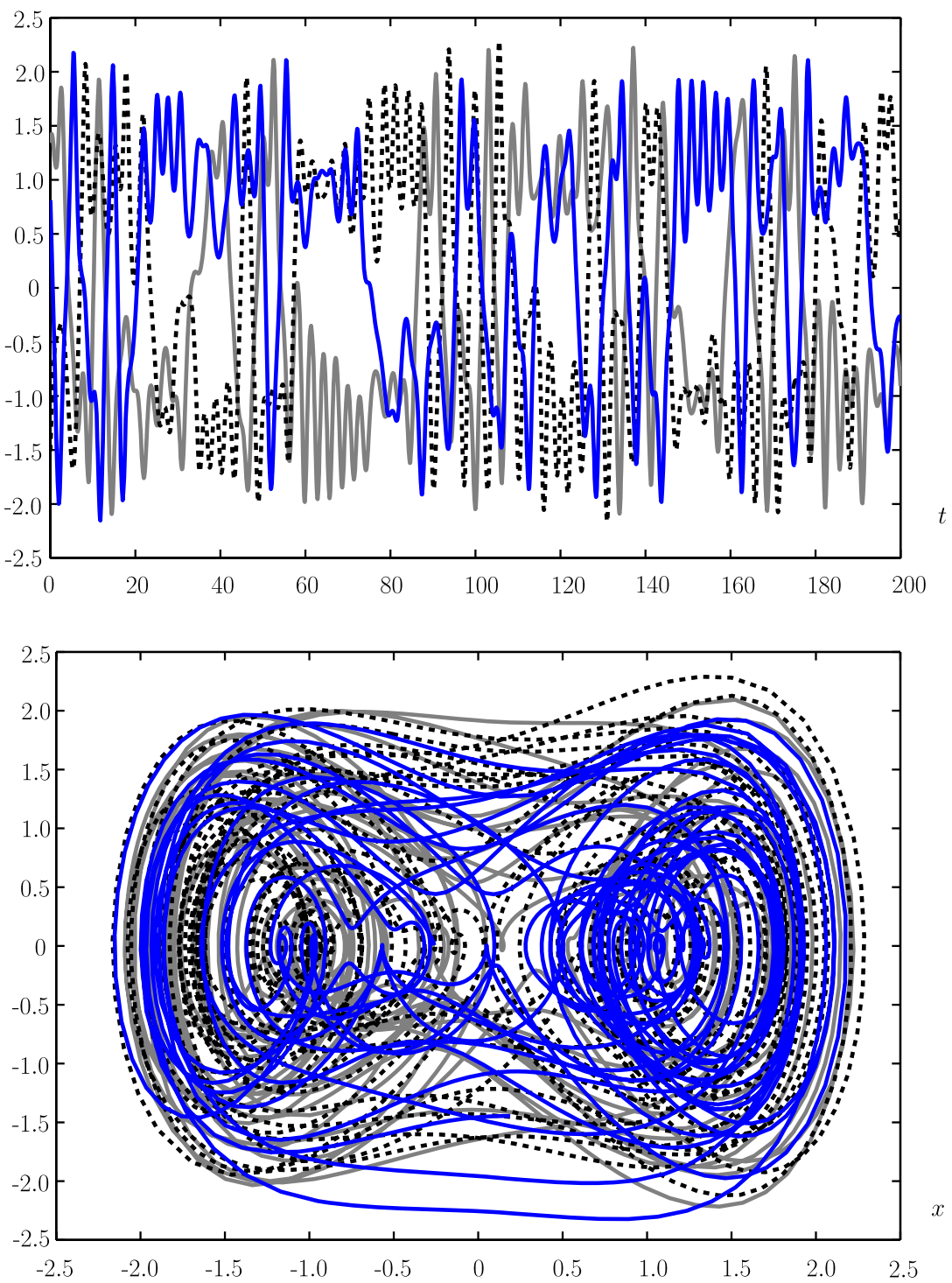

Table $4 N=3$ line symmetry classes

\begin{tabular}{lll}
\hline Label & Symmetry-related states & Stability \\
\hline$L_{1}$ & 000 & Unstable \\
$L_{2}$ & $A A A, B B B$ & Stable \\
$L_{3}$ & $A A 0,0 A A, B B 0,0 B B$ & Unstable \\
$L_{4}$ & $A A B, B A A, B B A, A B B$ & Stable \\
$L_{5}$ & $A 00,00 A, B 00,00 B$ & Unstable \\
$L_{6}$ & $A 0 B, B 0 A$ & Unstable \\
$L_{7}$ & $A 0 A, B 0 B$ & Unstable \\
$L_{8}$ & $A B A, B A B$ & Stable \\
$L_{9}$ & $A B 0, B A 0,0 B A, 0 A B$ & Unstable \\
$L_{10}$ & $0 A 0,0 B 0$ & Unstable \\
\hline
\end{tabular}

oscillator is out of phase. This is an example of broken symmetry due to coupling, since for small nonzero $c$, the permutation symmetry of the $A A$ pair is destroyed - the changing amplitude of the center oscillator prevents the $S_{N_{A}}$ permutation operators from mapping the solutions identically back to themselves. This class of solutions is unstable for small $|c|$.

The $L_{4}$ class is the set of solutions where the center oscillator is synchronized in a large periodic orbit with one of the end oscillators and the other oscillator has an anti-synchronized large periodic orbit. Similarly to the $L_{3}$ solutions, this class also has a bifurcation pattern that leaves one of the end oscillators unchanged in a larger periodic orbit. The center oscil- 
Fig. 18 Example of $L_{5}$ periodic orbit for $b=0.2$, $F=0.5, \omega_{f}=2.25$, $c=0.03$ with line topology. In the lower panel, the dark line, the light line, and the dashed line correspond to the three different oscillators
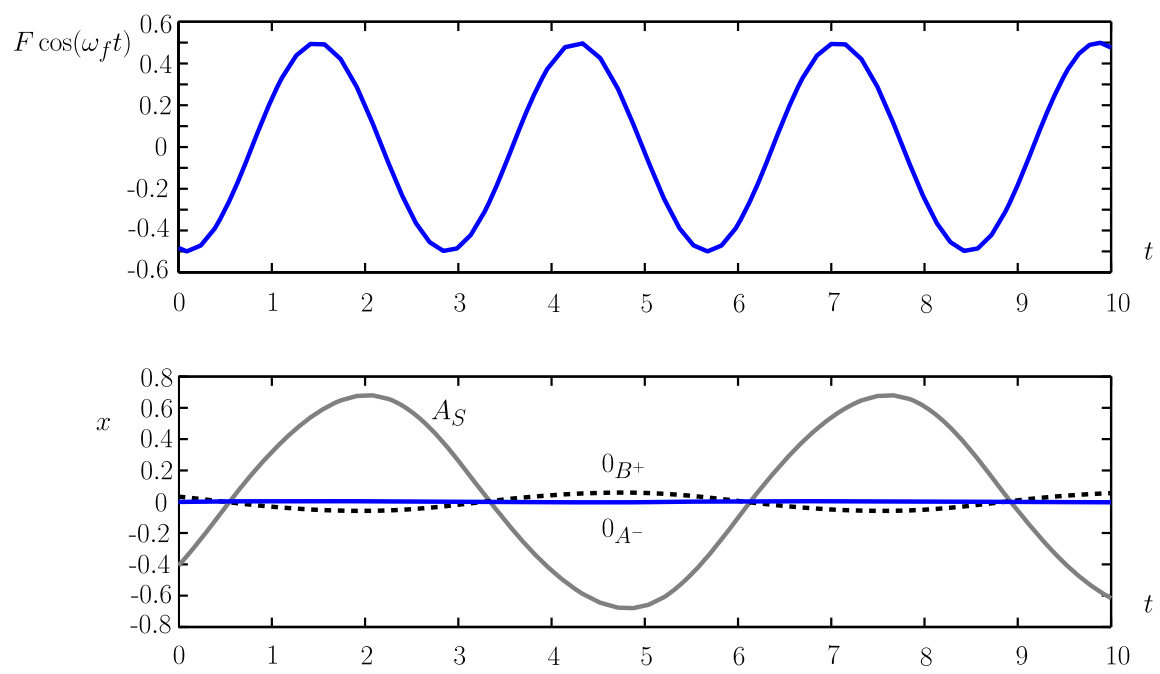

lator and the other end oscillator are identical but out of phase, and have larger magnitudes than the uncoupled case for negative coupling, and smaller magnitudes for positive coupling. Although the permutation symmetry between the $A$ solutions is broken by nonzero $c$, the symmetry between the center $A$ solution and the end $B$ solution remains for small $c$. The $L_{4}$ solutions are all stable for small $|c|$.

The $L_{5}$ class is the set of solutions where the center and one of the end oscillators are near-zero and the other end oscillator has a large periodic orbit. Symmetries in this class are completely broken for nonzero coupling. For $c \lesssim 0$, the large periodic orbit has larger magnitude than in the uncoupled system, and the two zero solutions become distinct near-zero periodic orbits in phase with the $A$ solution. A surprising behavior found numerically is that for small positive coupling, the zero solution of the center oscillator switches to be out of phase with respect to the other two oscillators, even though this phase relationship is not a mirror image of the negative coupling case. For both coupling cases, the amplitude of the center oscillator's near-zero orbit is slightly larger than that of the end oscillator's near-zero orbit. We show this solution in Fig. 18. These solutions are unstable for small $|c|$.

The $L_{6}$ class consists of the solutions where the center oscillator is the 0 solution and the two end oscillators have large periodic orbits that are in antisynchrony. The symmetry of the uncoupled case is preserved for small coupling for solutions in this class. When the coupling is negative, the oscillatory solutions are larger than the uncoupled case, and for posi-
Table 5 Solutions representatives for $|c| \approx 0$ for each symmetry class for line topology

\begin{tabular}{llll}
\hline Symmetry class & $c \lesssim 0$ & $c=0$ & $0 \lesssim c$ \\
\hline$L_{1}$ & $0_{0} 0_{0} 0_{0}$ & $0_{0} 0_{0} 0_{0}$ & $0_{0} 0_{0} 0_{0}$ \\
$L_{2}$ & $A_{0} A_{0} A_{0}$ & $A_{0} A_{0} A_{0}$ & $A_{0} A_{0} A_{0}$ \\
$L_{3}$ & $A_{0} A_{L} 0_{A}$ & $A_{0} A_{0} 0_{0}$ & $A_{0} A_{S} 0_{B}$ \\
$L_{4}$ & $A_{0} A_{L} B_{L}$ & $A_{0} A_{0} B_{0}$ & $A_{0} A_{S} B_{S}$ \\
$L_{5}$ & $A_{L} 0_{A^{+}} 0_{A^{-}}$ & $A_{0} 0_{0} 0_{0}$ & $A_{S} 0_{B^{+}} 0_{A^{-}}$ \\
$L_{6}$ & $A_{L} 0_{0} B_{L}$ & $A_{0} 0_{0} B_{0}$ & $A_{S} 0_{0} B_{S}$ \\
$L_{7}$ & $A_{L} 0_{A} A_{L}$ & $A_{0} 0_{0} A_{0}$ & $A_{S} 0_{B} A_{S}$ \\
$L_{8}$ & $A_{L^{-}} B_{L^{+}} A_{L^{-}}$ & $A_{0} B_{0} A_{0}$ & $A_{S^{+}} B_{S^{-}} A_{S^{+}}$ \\
$L_{9}$ & $A_{L^{-}} B_{L^{+}} 0_{B}$ & $A_{0} B_{0} 0_{0}$ & $A_{S^{+}} B_{S^{-}} 0_{A}$ \\
$L_{10}$ & $0_{A} A_{L} 0_{A}$ & $0_{0} A_{0} 0_{0}$ & $0_{B} A_{S} 0_{B}$ \\
\hline
\end{tabular}

tive coupling the reverse is true, as shown in Table 5. Interestingly, the center oscillator's zero solution remains identically zero. The $L_{6}$ solutions are unstable for small $|c|$.

The $L_{7}$ class is the set of solutions where the center oscillator is near-zero and the two end oscillators are synchronized with large periodic orbits. Solutions in this class also retain their symmetry for small nonzero coupling. For $c \lesssim 0$, the two end oscillators follow orbits that are slightly larger than the uncoupled $A$ solution, and the zero solution becomes a near-zero solution in phase with the other two oscillators. For $0 \lesssim c$, the magnitude of the end oscillators' larger periodic orbit becomes slightly smaller than the uncoupled case, and the near-zero solution is out of phase. These solutions are also unstable for small $|c|$. 
Fig. 19 Example of $L_{9}$ periodic orbit for $b=0.2$, $F=0.5, \omega_{f}=2.25$, $c=0.03$ with line topology. In the lower panel, the dark line, the light line, and the dashed line correspond to the three different oscillators
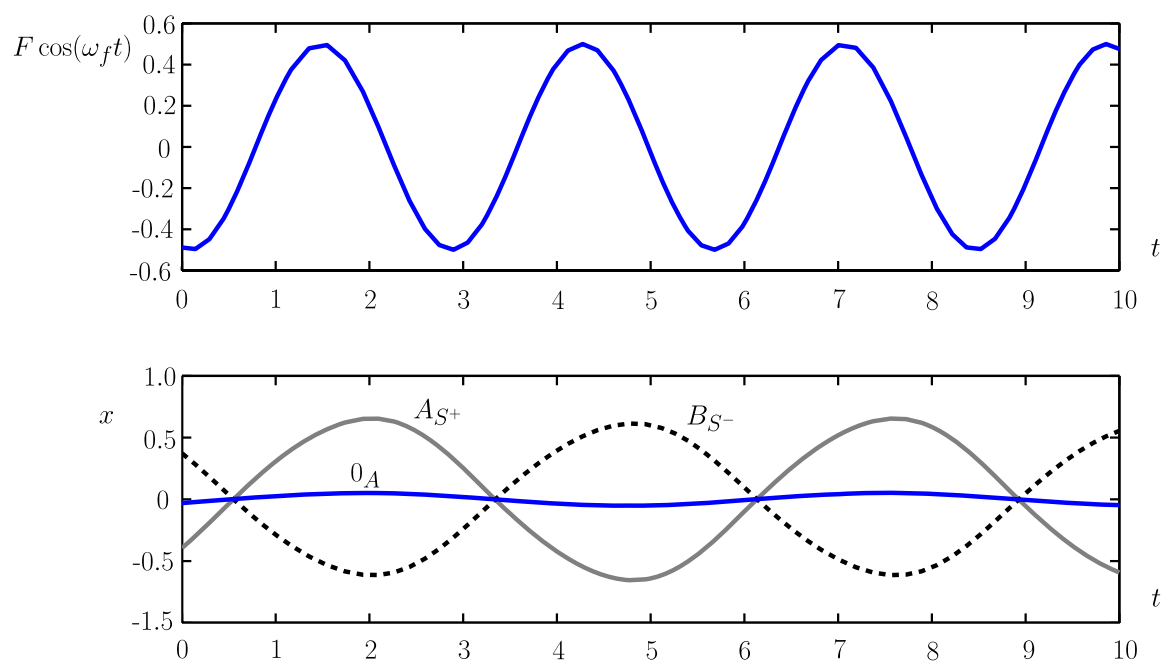

The $L_{8}$ class is the set of solutions where the two end oscillators have large synchronous periodic orbits in anti-synchrony with the center oscillator's large periodic orbit. Symmetries of solution trajectories in this class are modified by coupling in an interesting way. The two end oscillators retain their permutation symmetry, but the symmetries with the center oscillator are lost, because the amplitudes no longer match. When the small coupling is negative, the center oscillator has a larger orbit than the two end oscillators which themselves have a slightly larger orbit than the uncoupled case. The situation is reversed for the case of small positive coupling, as shown in Table 5. These two solutions are stable for small $|c|$.

The $L_{9}$ class is composed of the solutions for which the center oscillator has a large periodic orbit in antisynchrony with one of the end oscillators, and the other end oscillator has a near-zero orbit. The solution symmetries for this class are broken by coupling. For $c \lesssim 0$, the two non-zero solutions become larger than the uncoupled periodic orbit, but are not identical, and the zero solution becomes a near-zero solution in phase with the center oscillator. Small positive coupling yields a similar situation, with the two periodic solutions becoming slightly smaller than the uncoupled orbit, and non-identical, while the near-zero solution is in phase with the other end oscillator. This solution is shown in Fig. 19. The $L_{9}$ class of solutions are all unstable for small $|c|$.

The $L_{10}$ class is the set of solutions where the two end oscillators have near-zero orbits and the center oscillator has a large periodic orbit. This class of so- lutions retains a version of its uncoupled symmetry. For small negative coupling, the center oscillator has a slightly larger orbit than the uncoupled trajectory, and the two end oscillators follow identical synchronous near-zero orbits. The opposite is true for small positive coupling. This class of solutions is unstable for small $|c|$.

The properties for the solutions for $|c| \approx 0$ are summarized for reference in Table 5.

\subsubsection{Forcing-frequency Bifurcation diagram: Line topology}

We again fix the coupling strength as $c=0.03$ and take $b=0.2, F=0.5$, and treat $\omega_{f}$ as a bifurcation parameter. Figure 20 shows the $\max \left(\left|x_{1}\right|\right)$ projection of the corresponding bifurcation diagram and Fig. 21 shows the $\max \left(\left|x_{2}\right|\right)$-projection. We note that the $\max \left(\left|x_{1}\right|\right)$-projection is visually identical to the $\max \left(\left|x_{3}\right|\right)$-projection, as the two end oscillators (numbers 1 and 3 ) differ only by a label permutation. As in the previous bifurcation diagrams, the in-phase $A A A$ solution and the $A 0 B$ solution bifurcate from the 000 solution at different values of $\omega_{f}$. The structure of these bifurcation diagrams is similar to the $N=2$ case shown in Figs. 7 and 8. We see the large-small solution branches are substantially different in magnitude for the center (number 2) oscillator versus the end oscillators (numbers 1 and 3 ). 
Fig. $20 \quad N=3$ line topology bifurcation diagram for fixed $b=0.2$, $F=0.5, c=0.03$ with $\omega_{f}$ treated as a bifurcation parameter $\left(\max \left(\left|x_{1}\right|\right)\right.$-projection). Solid (resp., dashed) lines indicate stable (resp., unstable) solutions. Stable solutions are labeled by symmetry class per the $0 \lesssim c$ column of Table 5 , and have symmetry-related solutions tabulated in Table 4
Fig. $21 N=3$ line topology bifurcation diagram for fixed $b=0.2$, $F=0.5, c=0.03$ with $\omega_{f}$ treated as a bifurcation parameter

(max $\left(\left|x_{2}\right|\right)$-projection). Solid (resp., dashed) lines indicate stable (resp., unstable) solutions. Stable solutions are labeled by symmetry class per the $0 \lesssim c$ column of Table 5 , and have symmetry-related solutions tabulated in Table 4
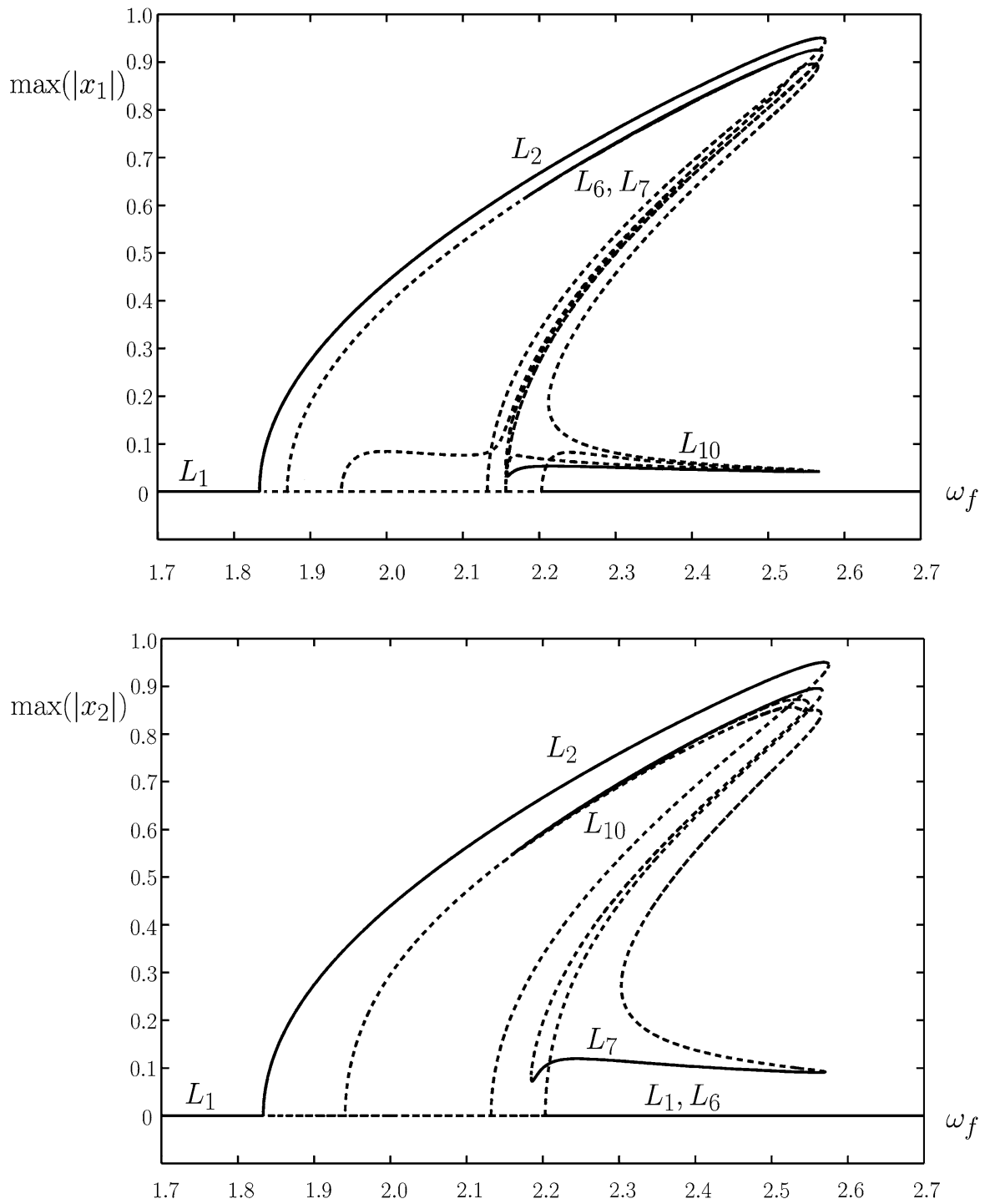

\subsubsection{Non-uniform coupling: Morphing from a line to a ring}

By starting with the ring topology, holding two of the coupling links fixed, and varying the third ( $\tilde{c}$ in (20)(22)), we can continuously morph the network from the ring topology to the line topology. In so doing, we can see how the symmetry classes change due to the topology.

The $R_{3}$ class splits into two classes, $L_{3}$ and $L_{7}$, the $R_{4}$ class splits into the $L_{4}$ and $L_{8}$ classes, the $R_{5}$ class splits into the $L_{5}$ and $L_{10}$ classes, and the $R_{6}$ class splits into the $L_{6}$ and $L_{9}$ classes. It is interesting to note that the stability properties of the split $L$ classes are inherited from the parent $R$ classes.

\section{$4.4 N$ coupled oscillators}

These results generalize to $N$ coupled parametrically forced oscillators. Specifically, provided the periodic orbits for the individual oscillators for the uncoupled system are hyperbolic, for every periodic solution which exists for the uncoupled system there will be a nearby periodic orbit for the system with sufficiently small coupling. Furthermore, the periodic orbit will inherit the stability properties from the periodic orbit for the uncoupled system. 
Notably, the Implicit Function Theorem argument presented above does not depend on the coupling topology of the system, or any special properties about the coupling strength (for example, all the strengths being equal). That is, regardless of how the oscillators are coupled together, for sufficiently small coupling strengths there will be analogs of the periodic solutions which exist for the uncoupled system. For example, instead of the oscillators having all-to-all coupling, similar results hold for oscillators coupled only to their neighbors. Of course, as the coupling strengths increase away from zero, so that the above arguments no longer hold, the coupling topology will affect the types of states that exist and are stable.

\section{Conclusion}

We have discussed periodic solutions which occur for parametrically forced oscillators that are weakly coupled together. A combinatorial approach was presented to enumerate all possible solutions for the uncoupled case. The existence and stability of periodic orbits for the coupled system can be determined by the existence and stability of the individual parametrically forced oscillators when they are uncoupled. Our results follow from an application of the Implicit Function Theorem to an appropriate Poincaré map. The results were confirmed using numerical bifurcation analysis for several simple networks of oscillators with 2:1 excitation resonance. An explicit expression was given for the isotropy subgroup of symmetric solutions, and the generators of those subgroups were identified. We classified the behavior of the solutions as a function of coupling strength, and classified the symmetry-breaking that occurs for non-zero coupling, which depends on network topology. We also explored the solution bifurcation structure as a function of forcing frequency for our example networks, which could be useful for experimental purposes, for example in MEMS research [8, 18, 26, 31, 34]. These results may be viewed as an analog of general results on the existence of phase-locked solutions for weakly coupled autonomous oscillators, as in $[1,6]$.

We remark that while it is instructive to study systems with symmetries as we have done in this paper, in real systems such symmetries will not be exact and symmetry breaking imperfections must be assumed to be present. For example, not all oscillators will be identical due to imprecision in their fabrication. Studies of systems with weakly broken symmetry have shown that analogues of the solutions when there is exact symmetry will persist; however, broken symmetries can lead to the appearance of new solutions which have no analogue in the fully symmetric system [21, 22]. The study of coupled oscillator systems with broken symmetry is deferred to future work.

Acknowledgements P.D. is supported by the National Science Foundation through the Integrative Graduate Education and Research Trainee-ship program. J.M. is supported by the National Science Foundation under Grants NSF-0428916 and NSF-0547606. We thank Jeff Rhoads and Eric Shea-Brown for helpful discussions related to this work.

Open Access This article is distributed under the terms of the Creative Commons Attribution Noncommercial License which permits any noncommercial use, distribution, and reproduction in any medium, provided the original author(s) and source are credited.

\section{Appendix: The Implicit Function Theorem}

We state the Implicit Function Theorem, adapted from [16].

Let $\Phi: \mathbb{R}^{N} \rightarrow \mathbb{R}^{M}, 1 \leq M<N$, be a continuously differentiable function, written as

$$
\begin{aligned}
\Phi(\mathbf{x}) & =\Phi\left(x_{1}, \ldots, x_{N}\right) \\
& =\left(\phi_{1}\left(x_{1}, \ldots, x_{N}\right), \ldots, \phi_{M}\left(x_{1}, \ldots, x_{N}\right)\right) .
\end{aligned}
$$

Suppose

$\Phi\left(\mathbf{x}^{0}\right)=\Phi\left(x_{1}^{0}, \ldots, x_{N}^{0}\right)=0$,

and

$\operatorname{det}\left(\begin{array}{cccc}\left.\frac{\partial \phi_{1}}{\partial x_{N-M+1}}\right|_{\mathbf{x}^{0}} & \left.\frac{\partial \phi_{1}}{\partial x_{N-M+2}}\right|_{\mathbf{x}^{0}} & \cdots & \left.\frac{\partial \phi_{1}}{\partial x_{N}}\right|_{\mathbf{x}^{0}} \\ \left.\frac{\partial \phi_{2}}{\partial x_{N-M+1}}\right|_{\mathbf{x}^{0}} & \left.\frac{\partial \phi_{2}}{\partial x_{N-M+2}}\right|_{\mathbf{x}^{0}} & \cdots & \left.\frac{\partial \phi_{2}}{\partial x_{N}}\right|_{\mathbf{x}^{0}} \\ \vdots & \vdots & & \vdots \\ \left.\frac{\partial \phi_{M}}{\partial x_{N-M+1}}\right|_{\mathbf{x}^{0}} & \left.\frac{\partial \phi_{M}}{\partial x_{N-M+2}}\right|_{\mathbf{x}^{0}} & \cdots & \left.\frac{\partial \phi_{M}}{\partial x_{N}}\right|_{\mathbf{x}^{0}}\end{array}\right) \neq 0$.

Then there exists a unique continuously differentiable function $f=\left(f_{1}, \ldots, f_{M}\right)$ from a neighborhood of $\left(x_{1}^{0}, \ldots, x_{N-M}^{0}\right) \in \mathbb{R}^{N-M}$ to a neighborhood of $\left(x_{N-M+1}^{0}, \ldots, x_{N}^{0}\right) \in \mathbb{R}^{M}$ such that

$$
\begin{gathered}
\Phi\left(x_{1}, \ldots, x_{N-M}, f_{1}\left(x_{1}, \ldots, x_{N-M}\right), \ldots,\right. \\
\left.f_{M}\left(x_{1}, \ldots, x_{N-M}\right)\right)=0 .
\end{gathered}
$$




\section{References}

1. Ashwin, P., Swift, J.: The dynamics of $n$ weakly coupled identical oscillators. J. Nonlinear Sci. 2, 69-108 (1992)

2. Bena, I., Van den Broeck, C.: Coupled parametric oscillators. Europhys. Lett. 48, 498-504 (1999)

3. Bena, I., Van den Broeck, C., Kawai, R., Copelli, M., Lindenberg, K.: Collective behavior of parametric oscillators. Phys. Rev. E 65, 036,611 (2002)

4. Bennett, M., Schatz, M.F., Rockwood, H., Wiesenfeld, K.: Huygen's clocks. Proc. R. Soc. Lond. A 458, 563-579 (2002)

5. Bromberg, Y., Cross, M.C., Lifshitz, R.: Response of discrete nonlinear systems with many degrees of freedom. Phys. Rev. E 73, 016214 (2006)

6. Brown, E., Holmes, P., Moehlis, J.: Globally coupled oscillator networks. In: Kaplan, E., Marsden, J., Sreenivasan, K. (eds.) Perspectives and Problems in Nonlinear Science: A Celebratory Volume in Honor of Larry Sirovich, pp. 183-215. Springer, New York (2003)

7. Brown, E., Moehlis, J., Holmes, P.: On the phase reduction and response dynamics of neural oscillator populations. Neural Comp. 16, 673-715 (2004)

8. Buks, E., Roukes, M.L.: Electrically tunable collective response in a coupled micromechanical array. J. MEMS 11, 802-807 (2002)

9. Daido, H.: Generic scaling at the onset of macroscopic mutual entrainment in limit cycles with uniform all-to-all coupling. Phys. Rev. Lett. 73, 760-763 (1994)

10. Doedel, E., Champneys, A., Fairgrieve, T., Kuznetsov, Y., Sandstede, B., Wang, X.: AUTO 97: Continuation and bifurcation software for ordinary differential equations. http://indy.cs.concordia.ca/auto/ (1997)

11. Ermentrout, G.B.: Simulating, Analyzing, and Animating Dynamical Systems: A Guide to XPPAUT for Researchers and Students. SIAM, Philadelphia (2002)

12. Goldobin, D., Pikovsky, A.: Collective modes in parametrically excited oscillator arrays. Europhys. Lett. 59, 193-198 (2002)

13. Guckenheimer, J.: Isochrons and phaseless sets. J. Math. Biol. 1, 259-273 (1975)

14. Guckenheimer, J., Holmes, P.J.: Nonlinear Oscillations, Dynamical Systems and Bifurcations of Vector Fields. Springer, New York (1983)

15. Huygens, C.: Instructions concerning the use of pendulumwatches for finding the longitude at sea. Philos. Trans. R. Soc. Lond. 4, 937 (1669)

16. Krantz, S.G., Parks, H.R.: The Implicit Function Theorem: History, Theory, and Applications. Birkhäuser, Boston (2002)

17. Kuramoto, Y.: Chemical Oscillations, Waves, and Turbulence. Springer, Berlin (1984)
18. Lifshitz, R., Cross, M.C.: Response of parametrically driven nonlinear coupled oscillators with application to micromechanical and nanomechanical resonator arrays. Phys. Rev. B 67, 134302 (2003)

19. Michaels, D.C., Matyas, E.P., Jalife, J.: Mechanisms of sinoatrial pacemaker synchronization-a new hypothesis. Circ. Res. 61, 704-714 (1987)

20. Moehlis, J.: On the dynamics of coupled parametrically forced oscillators. In: Proceedings of 2008 ASME Dynamic Systems and Control Conference. Ann Arbor, Michigan, USA, October 20-22, 2008. DSCC2008-2189

21. Moehlis, J., Knobloch, E.: Forced symmetry breaking as a mechanism for bursting. Phys. Rev. Lett. 80, 5329-5332 (1998)

22. Moehlis, J., Knobloch, E.: Bursts in oscillatory systems with broken $\mathrm{D}_{4}$ symmetry. Physica D 135, 263-304 (2000)

23. Nayfeh, A.H., Mook, D.T.: Nonlinear Oscillations. Wiley, New York (1979)

24. Okuda, K.: Variety and generality of clustering in globally coupled oscillators. Physica D 63, 424-436 (1993)

25. Pikovsky, A., Rosenblum, M., Kurths, J.: Synchronization: A Universal Concept in Nonlinear Sciences. Cambridge University Press, Cambridge (2001)

26. Rhoads, J.F., Shaw, S.W., Turner, K.L., Moehlis, J., DeMartini, B.E., Zhang, W.: Generalized parametric resonance in electrostatically actuated microelectromechanical oscillators. J. Sound Vib. 296, 797-829 (2006)

27. Strogatz, S.H.: From Kuramoto to Crawford: Exploring the onset of synchronization in populations of coupled oscillators. Physica D 143, 1-20 (2000)

28. Strogatz, S.: Sync: The Emerging Science of Spontaneous Order. Hyperion, New York (2003)

29. Tezak, E.G., Nayfeh, A.H., Mook, D.T.: Parametrically excited non-linear multidegree-of-freedom systems with repeated natural frequencies. J. Sound. Vib. 85, 459-472 (1982)

30. Traub, R.D., Miles, R., Wong, R.K.S.: Model of the origin of rhythmic population oscillations in the hippocampal slice. Science 243, 1319-1325 (1989)

31. Turner, K.L., Miller, S.A., Hartwell, P.G., MacDonald, N.C., Strogatz, S.H., Adams, S.G.: Five parametric resonances in a microelectromechanical system. Nature 396, 149-152 (1998)

32. Wiggins, S.: Introduction to Applied Nonlinear Dynamical Systems and Chaos, 2nd edn. Springer, New York (2003)

33. Winfree, A.: The Geometry of Biological Time, 2nd edn. Springer, New York (2001)

34. Zhang, W., Baskaran, R., Turner, K.: Tuning the dynamic behavior of parametric resonance in a micromechanical oscillator. Appl. Phys. Lett. 82, 130-132 (2003) 